Épijournal de Géométrie Algébrique

epiga.episciences.org

Volume 1 (2017), Article Nr. 4

\title{
Some remarks on regular foliations with numerically trivial canonical class
}

\author{
Stéphane Druel
}

\begin{abstract}
In this article, we first describe codimension two regular foliations with numerically trivial canonical class on complex projective manifolds whose canonical class is not numerically effective. Building on a recent algebraicity criterion for leaves of algebraic foliations, we then address regular foliations of small rank with numerically trivial canonical class on complex projective manifolds whose canonical class is pseudo-effective. Finally, we confirm the generalized Bondal conjecture formulated by Beauville in some special cases.
\end{abstract}

Keywords. Foliation

2010 Mathematics Subject Classification. 37F75

[Français]

Titre. Quelques remarques sur les feuilletages réguliers de classe canonique numériquement triviale

Résumé. Dans cet article nous décrivons tout d'abord les feuilletages de codimension deux de classe canonique numériquement triviale sur des variétés projectives complexes dont la classe canonique n'est pas numériquement effective. En nous appuyant sur un critère d'algébricité récent pour les feuilles des feuilletages algébriques, nous considérons ensuite les feuilletages réguliers de petit rang et de classe canonique numériquement triviale sur des variétés projectives complexes dont la classe canonique est pseudo-effective. Finalement, nous confirmons, dans certains cas spécifiques, la conjecture de Bondal généralisée formulée par Beauville.

Received by the Editors on October 5, 2016, and in final form on April 7, 2017. Accepted on May 12, 2017.

Stéphane Druel

Institut Fourier, UMR 5582 du CNRS, Université Grenoble Alpes, CS 40700, 38058 Grenoble cedex 9, France e-mail: stephane.druel@univ-grenoble-alpes.fr

The author was partially supported by the ALKAGE project (ERC grant Nr 670846, 2015-2020).

(C) by the author(s)

This work is licensed under http://creativecommons.org/licenses/by-sa/4.0/ 


\section{Contents}

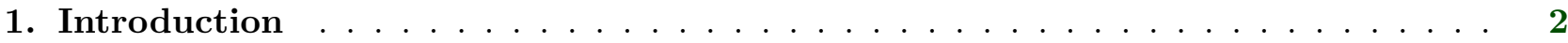

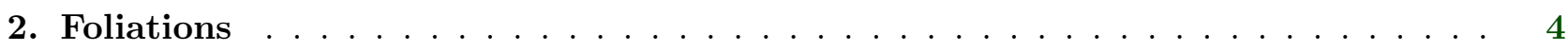

3. Bott (partial) connection and applications $\ldots \ldots \ldots \ldots$

4. Projectable foliations under Fano-Mori contractions $\ldots \ldots \ldots \ldots$

5. Codimension 2 (regular) foliations with numerically trivial canonical class . . . 11

6. Algebraically integrable regular foliations with numerically trivial canonical class 12

7. Poisson manifolds - Generalized Bondal conjecture $\ldots \ldots \ldots \ldots$

\section{Introduction}

The Beauville-Bogomolov decomposition theorem asserts that any compact Kähler manifold with numerically trivial canonical bundle admits an étale cover that decomposes into a product of a torus, and irreducible, simply-connected Calabi-Yau, and holomorphic symplectic manifolds (see [Bea83]). In [Tou08], Touzet obtained a foliated version of the Beauville-Bogomolov decomposition theorem for codimension 1 regular foliations with numerically trivial canonical bundle on compact Kähler manifolds. The next statement follows from [Tou08, Théorème 1.2] and [Dru16, Lemma 5.9].

Theorem (Touzet). Let $X$ be a complex projective manifold, and let $\mathscr{G} \subset T_{X}$ be a regular codimension 1 foliation on $X$ with $K_{\mathscr{G}} \equiv 0$. Then one of the following holds.

(1) There exists a $\mathbb{P}^{1}$-bundle structure $\varphi: X \rightarrow Y$ onto a complex projective manifold $Y$ with $K_{Y} \equiv 0$, and $\mathscr{G}$ induces a flat holomorphic connection on $\varphi$.

(2) There exists an abelian variety $A$ as well as a simply connected projective manifold $Y$ with $K_{Y} \equiv 0$, and a finite étale cover $f: A \times Y \rightarrow X$ such that $f^{-1} \mathscr{G}$ is the pull-back of a codimenion 1 linear foliation on $A$.

(3) There exists a smooth complete curve $B$ of genus at least 2 as well as a complex projective manifold $Y$ with $K_{Y} \equiv 0$, and a finite étale cover $f: B \times Y \rightarrow X$ such that $f^{-1} \mathscr{G}$ is induced by the projection morphism $B \times Y \rightarrow B$.

Pereira and Touzet addressed regular foliations $\mathscr{G}$ on complex projective manifolds with $c_{1}(\mathscr{G}) \equiv 0$ and $c_{2}(\mathscr{G}) \equiv 0$ in [PT13]. They show the following (see [PT13, Theorem C]).

Theorem (Pereira-Touzet). Let $X$ be a complex projective manifold, and let $\mathscr{G} \subset T_{X}$ be a regular foliation on $X$ with $c_{1}(\mathscr{G}) \equiv 0$ and $c_{2}(\mathscr{G}) \equiv 0$. Then, there exists a finite étale cover $f: Z \rightarrow X$ as well as a dense open subset $Z^{\circ} \subset Z$, and a proper morphism $\varphi^{\circ}: Z^{\circ} \rightarrow Y^{\circ}$ whose fibers are abelian varieties such that $f^{-1} \mathscr{G}_{\mid Z^{\circ}} \subset T_{Z^{\circ} / Y^{\circ}}$ and such that $f^{-1} \mathscr{G}_{\mid Z^{\circ}}$ induces linear foliations on the fibers of $\varphi^{\circ}$.

They also show by way of example that $\varphi^{\circ}$ may not be a locally trivial fibration for the Euclidean topology (see [PT13, Example 4.2]). On the other hand, this phenomenon does not occur if $\mathscr{G}$ has codimension $\leqslant 2$. More precisely, they prove the following (see [PT13, Theorem D]). 
Theorem (Pereira-Touzet). Let $X$ be a complex projective manifold, and let $\mathscr{G} \subset T_{X}$ be a regular codimension 2 foliation on $X$ with $c_{1}(\mathscr{G}) \equiv 0$ and $c_{2}(\mathscr{G}) \equiv 0$. Then, there exists an abelian variety $A$ as well as a complex projective manifold $Y$, and a finite étale cover $f: A \times Y \rightarrow X$ such that $f^{-1} \mathscr{G}$ is the pull-back of a linear foliation on $A$.

In this article, we first address regular codimension 2 foliations with numerically trivial canonical class on complex projective manifolds $X$ with $K_{X}$ not nef.

Theorem 1.1. Let $X$ be a complex projective manifold, and let $\mathscr{G} \subset T_{X}$ be a regular codimension 2 foliation on $X$ with $K_{\mathscr{G}} \equiv 0$. Suppose that $K_{X}$ is not nef. Then one of the following holds.

(1) There exist a complex projective manifold $Y$, a regular codimension 2 foliation $\mathscr{G}_{Y}$ on $Y$ with $K_{\mathscr{G}_{Y}} \equiv 0$, and a compact leaf $Z \subset Y$ of $\mathscr{G}_{Y}$ such that $X$ is the blow-up of $Y$ along $Z$. Moreover, $\mathscr{G}$ is the pull-back of $\mathscr{G}_{Y}$.

(2) There exists a conic bundle structure $\varphi: X \rightarrow Y$ on $X$ onto a complex projective manifold $Y$, $\mathscr{G}$ is generically transverse to $\varphi$ and induces a regular codimension 1 foliation $\mathscr{G}_{Y}$ on $Y$ with $K_{\mathscr{G}_{Y}} \equiv 0$. Moreover, the discriminant locus of $\varphi$ is either empty or a union of leaves of $\mathscr{G}_{Y}$.

(3) There exists a smooth morphism $\varphi: X \rightarrow Y$ onto a complex projective manifold $Y$ of dimension $\operatorname{dim} Y=\operatorname{dim} X-2$ with $K_{Y} \equiv 0$, and $\mathscr{G}$ yields a flat holomorphic connection on $\varphi$.

In [PT13], the authors show that if $\mathscr{G}$ is a regular foliation with $c_{1}(\mathscr{G}) \equiv 0$ on a complex projective manifold $X$ with $K_{X}$ pseudo-effective, then $\mathscr{G}$ is polystable with respect to any polarization. They also conjecture that if moreover $\mathscr{G}$ is stable with respect to some polarization and $c_{2}(\mathscr{G}) \not \equiv 0$, then $\mathscr{G}$ is algebraically integrable (see [PT13, Section 6.5]). We recently confirmed this conjecture in some special cases in [Dru16]. Building on these results, we obtain the following.

Theorem 1.2. Let $X$ be a complex projective manifold, and let $\mathscr{G} \subset T_{X}$ be a regular foliation on $X$ with $K_{\mathscr{G}} \equiv 0$. Suppose that $K_{X}$ is pseudo-effective. Suppose furthermore that $\mathscr{G}$ has rank at most 3. Then there exist complex projective manifolds $Y$ and $F$, a finite étale cover $f: Y \times F \rightarrow X$, and a regular foliation $\mathscr{H}$ on $Y$ with $c_{1}(\mathscr{H}) \equiv 0$ and $c_{2}(\mathscr{H}) \equiv 0$ such that $f^{-1} \mathscr{G}$ is the pull-back of $\mathscr{H}$.

Notice that Touzet addressed codimension 2 regular foliations $\mathscr{G}$ with $c_{1}(\mathscr{G}) \equiv 0$ on compact Kähler manifolds with seminegative Ricci curvature in [Tou10]. See [Tou10, Théorème 1.5] for a precise statement.

Finally, we confirm the generalized Bondal conjecture formulated by Beauville ([Bea11, Conjecture 5]) in some special cases. Recall that a holomorphic Poisson structure on a complex manifold $X$ is a bivector field $\tau \in H^{0}\left(X, \wedge^{2} T_{X}\right)$, such that the bracket $\{f, g\}:=\langle\tau, d f \wedge d g\rangle$ defines a Lie algebra structure on $\mathscr{O}_{X}$. A Poisson structure defines a skew-symmetric map $\tau^{\sharp}: \Omega_{X}^{1} \rightarrow T_{X}$; the rank of $\tau$ at a point $x \in X$ is the rank of $\tau^{\sharp}(x)$. Let $r$ be the generic rank of $\tau$, and set $X_{r}:=\left\{x \in X \mid \operatorname{rk} \tau^{\sharp}(x)=r\right\}$.

Theorem 1.3. Let $(X, \tau)$ be a projective Poisson manifold. Suppose that $\tau$ has generic rank $r \geqslant$ $\operatorname{dim} X-2$. If the degeneracy locus $X \backslash X_{r}$ of $\tau$ is non-empty, then it has a component of dimension $>r-2$.

Outline of the paper. In section 2, we review basic definitions and results about foliations. Sections 3 and 4 consist of technical preparations. In section 3, we review standard facts about (partial) connections and give some applications. Section 4 presents a criterion that guarantees that a given foliation is projectable under a morphism. We then study projectable foliations under some Fano-Mori contractions. With these preparations at hand, the proof of Theorem 1.1 which we give in Section 5 
becomes reasonably short. Section 6 is devoted to the proof of Theorem 1.2. The proof relies on a global version of Reeb stability theorem for regular foliations with numerically trivial canonical class on complex projective manifolds with pseudo-effective canonical divisor. Finally, we prove Theorem 1.3 in section 7 .

Notation and global conventions. We work over the field $\mathbb{C}$ of complex numbers. Varieties are always assumed to be irreducible. We denote by $X_{\text {reg }}$ the smooth locus of a variety $X$.

The word "stable" will always mean "slope-stable with respect to a given polarization". Ditto for semistability.

Acknowledgements. We would like to thank Pierre-Emmanuel Chaput, Jorge V. Pereira and Frédéric Touzet for helpful discussions. We also thank the referee for their thoughtful suggestions on how to improve the presentation of some of the results.

\section{Foliations}

In this section we recall basic facts concerning foliations.

2.1. (Foliations) A foliation on a normal variety $X$ is a coherent subsheaf $\mathscr{G} \subset T_{X}$ such that

- $\mathscr{G}$ is closed under the Lie bracket, and

- $\mathscr{G}$ is saturated in $T_{X}$. In other words, the quotient $T_{X} / \mathscr{G}$ is torsion free.

The rank $r$ of $\mathscr{G}$ is the generic rank of $\mathscr{G}$. The codimension of $\mathscr{G}$ is defined as $q:=\operatorname{dim} X-r$. Let $U \subset X_{\text {reg }}$ be the open set where $\mathscr{G}_{\mid X_{\mathrm{reg}}}$ is a subbundle of $T_{X_{\mathrm{reg}}}$. The singular locus of $\mathscr{G}$ is defined as $Z(\mathscr{G}):=X \backslash U$. We say that $\mathscr{G}$ is regular if $U=X_{\text {reg }}=X$.

A leaf of $\mathscr{G}$ is a connected, locally closed holomorphic submanifold $L \subset U$ such that $T_{L}=\mathscr{G}_{\mid L}$. A leaf is called algebraic if it is open in its Zariski closure.

The canonical class $K_{\mathscr{G}}$ of $\mathscr{G}$ is any Weil divisor on $X$ such that $\mathscr{O}_{X}\left(-K_{\mathscr{G}}\right) \cong \operatorname{det}(\mathscr{G}):=\left(\wedge^{r} \mathscr{G}\right)^{* *}$.

We will use the following notation.

Notation 2.2. Let $\varphi: X \rightarrow Y$ be a dominant morphism of normal varieties, and let $D$ be a Weil $\mathbb{Q}$-divisor on $Y$. Suppose that $\varphi$ is equidimensional. The pull-back $\varphi^{*} D$ of $D$ is defined as follows. We define $\varphi^{*} D$ to be the unique $\mathbb{Q}$-divisor on $X$ whose restriction to $\varphi^{-1}\left(Y_{\text {reg }}\right)$ is $\left(\varphi_{\mid Y_{\text {reg }}}\right)^{*} D_{\mid Y_{\text {reg }}}$. This construction agrees with the usual pull-back if $D$ itself is $\mathbb{Q}$-Cartier.

Notation 2.3. Let $\varphi: X \rightarrow Y$ be a dominant morphism of normal varieties. Suppose that $\varphi$ is equidimensional. Write $K_{X / Y}:=K_{X}-\varphi^{*} K_{Y}$. We refer to it as the relative canonical divisor of $X$ over $Y$. Set $R(\varphi)=\sum_{D}\left((\varphi)^{*} D-\left((\varphi)^{*} D\right)_{\text {red }}\right)$ where $D$ runs through all prime divisors on $Y$. We refer to it as the ramification divisor of $\varphi$.

Example 2.4. Let $\varphi: X \rightarrow Y$ be a dominant morphism of normal varieties. Suppose that $\varphi$ is equidimensional, and let $\mathscr{G}$ be the foliation on $X$ induced by $\varphi$. A straightforward computation shows that $K_{\mathscr{G}} \sim K_{X / Y}-R(\varphi)$, where $R(\varphi)$ denotes the ramification divisor of $\varphi$.

2.5. (Foliations described as pullbacks) Let $X$ and $Y$ be normal varieties, and let $\varphi: X \rightarrow Y$ be a dominant morphism. Let $\mathscr{G}$ be a foliation on $Y$. The pullback $\varphi^{-1} \mathscr{G}$ of $\mathscr{G}$ under $\varphi$ is defined as follows. We define $\varphi^{-1} \mathscr{G}$ to be the unique foliation on $X$ whose restriction to $\varphi^{-1}\left(Y_{\text {reg }}\right)$ is the saturation of

$$
\left(d\left(\varphi_{\mid \varphi^{-1}\left(Y_{\mathrm{reg}}\right)}\right)\right)^{-1}\left(\left(\varphi_{\mid \varphi^{-1}\left(Y_{\mathrm{reg}}\right)}\right)^{*} \mathscr{G}_{\mid Y_{\mathrm{reg}}}\right)
$$

in $T_{X \mid \varphi^{-1}\left(Y_{\text {reg }}\right)}$, where $d\left(\varphi_{\mid \varphi^{-1}\left(Y_{\text {reg }}\right)}\right): T_{X \mid \varphi^{-1}\left(Y_{\text {reg }}\right)} \rightarrow\left(\varphi_{\mid \varphi^{-1}\left(Y_{\text {reg }}\right)}\right)^{*} T_{Y_{\text {reg }}}$ denotes the natural map. 
A straightforward computation shows the following.

Lemma 2.6. Let $f: Z \rightarrow X$ be a finite cover of normal varieties, and let $\mathscr{G}$ be a foliation on $X$. Suppose that the branch locus of $f$ is $\mathscr{G}$-invariant. Then $f^{-1} \mathscr{G} \cong\left(f^{*} \mathscr{G}\right)^{* *}$.

2.7. (Projection of foliations) Let $X$ and $Y$ be complex manifolds, and let $\mathscr{G} \subset T_{X}$ be a foliation of rank $r$ on $X$. Let also $\varphi: X \rightarrow Y$ be a dominant morphism. We say that $\mathscr{G}$ is projectable under $\varphi$ if, for a general point $y \in Y, d_{x} \varphi\left(\mathscr{G}_{x}\right)$ is independent of the choice of $x \in \varphi^{-1}(y)$ and $\operatorname{dim} d_{x} \varphi\left(\mathscr{G}_{x}\right)=r$, where $d_{x} \varphi: T_{x} X \rightarrow T_{y} Y$ denotes the natural map. We claim that $\mathscr{G}$ induces a foliation $\mathscr{G}_{Y}$ of rank $r$ on $Y$. Indeed, we may assume without loss of generality that $\varphi$ is smooth. Let $y \in Y$ be a general point, and let $s: W \rightarrow X$ be a section of $\varphi$ over some open neighborhood $W$ of $y$ with respect to the Euclidean topology. Since $\mathscr{G}$ is projectable, $\mathscr{G}_{\mid s(W)} \subset T_{s(W)} \subset\left(T_{X}\right)_{\mid s(W)}$, and we must have $\mathscr{G}_{\mid \varphi^{-1}(W)} \cong\left(\varphi_{\mid \varphi^{-1}(W)}\right)^{*}\left(s^{*} \mathscr{G}\right)$. This immediately implies that $s^{*} \mathscr{G} \subset T_{W}$ is independent of $s$, and that it is stable under the Lie bracket. Let $\mathscr{G}_{Y}$ be the foliation on $Y$ such that $\left(\mathscr{G}_{Y}\right)_{\mid W}:=s^{*} \mathscr{G}$. We will refer to $\mathscr{G}_{Y}$ as the projection of $\mathscr{G}$ under $\varphi$.

Suppose from now on that $\varphi$ is a proper morphism with connected fibers. Suppose furthermore that $\operatorname{det}(\mathscr{G}) \cong \varphi^{*} \mathscr{L}$ for some line bundle $\mathscr{L}$ on $Y$.

The $r$-th wedge product of the inclusion $\mathscr{G} \subset T_{X}$ gives rise to a non-zero global section $\tau \in$ $H^{0}\left(X, \wedge^{r} T_{X} \otimes \varphi^{*} \mathscr{L}^{*}\right)$ mapping to a possibly zero global section $\tau_{Y} \in H^{0}\left(Y, \wedge^{r} T_{Y} \otimes \mathscr{L}^{*}\right)$ under the natural map

$$
H^{0}\left(X, \wedge^{r} T_{X} \otimes \varphi^{*} \mathscr{L}^{*}\right) \longrightarrow H^{0}\left(X, \wedge^{r}\left(\varphi^{*} T_{Y}\right) \otimes \varphi^{*} \mathscr{L}^{*}\right) \cong H^{0}\left(Y, \wedge^{r} T_{Y} \otimes \mathscr{L}^{*}\right) .
$$

Under these assumptions, $\mathscr{G}$ is projectable under $\varphi$ if and only if $\tau_{Y} \neq 0$. Equivalently, $\mathscr{G}$ is projectable under $\varphi$ if and only if there exists $x \in X$ such that $\operatorname{dim} d_{x} \varphi\left(\mathscr{G}_{x}\right)=r$. Let $Z\left(\tau_{Y}\right)$ be the zero set of $\tau_{Y}$. Then $\varphi(Z(\mathscr{G})) \subset Z\left(\tau_{Y}\right)$, and conversely, if $x \in X \backslash Z(\mathscr{G})$ and $\operatorname{dim} d_{x} \varphi\left(\mathscr{G}_{x}\right)=r$, then $\varphi(x) \in Y \backslash Z\left(\tau_{Y}\right)$.

Finally, suppose that $\tau_{Y} \neq 0$, and let $\mathscr{G}_{Y}$ be the projection of $\mathscr{G}$ under $\varphi$. We claim that $-K \mathscr{G}_{Y}-$ $c_{1}(\mathscr{L})$ is effective. Indeed, let $U \subset Y$ be the Zariski open set where $\mathscr{G}_{Y}$ is a subbundle of $T_{Y}$. The natural map $d \varphi: T_{X} \rightarrow \varphi^{*} T_{Y}$ induces a morphism $\mathscr{G}_{\mid \varphi^{-1}(U)} \rightarrow\left(\varphi_{\mid \varphi^{-1}(U)}\right)^{*}\left(\mathscr{G}_{Y}\right)_{\mid U}$ which is generically injective. In particular, we must have $\left(\tau_{Y}\right)_{\mid U} \in H^{0}\left(U,\left(\operatorname{det}\left(\mathscr{G}_{Y}\right) \otimes \mathscr{L}^{*}\right)_{\mid U}\right)$. Since $Y \backslash U$ has codimension at least two, we conclude that $-K_{\mathscr{G}_{Y}}-c_{1}(\mathscr{L})$ is effective, proving our claim. Suppose moreover that $-K_{\mathscr{G}_{Y}} \equiv c_{1}(\mathscr{L})$. Then $\left(\operatorname{det}\left(\mathscr{G}_{Y}\right) \otimes \mathscr{L}^{*}\right)_{\mid U} \cong \mathscr{O}_{U}$ and $Z\left(\tau_{Y}\right) \subset Y \backslash U$. It follows that $Z\left(\tau_{Y}\right)$ has codimension at least two, and hence $Z\left(\mathscr{G}_{Y}\right)=Z\left(\tau_{Y}\right)$. In particular, we must have $\varphi(Z(\mathscr{G})) \subset$ $Z\left(\tau_{Y}\right)=Z\left(\mathscr{G}_{Y}\right)$.

Remark 2.8. Let $X$ be a complex projective manifold, and let $\mathscr{G} \subset T_{X}$ be a foliation on $X$. Let also $\varphi: X \rightarrow Y$ be a proper morphism with connected fibers onto a normal projective variety $Y$. Suppose that $-K_{X}$ is $\varphi$-ample. If $K_{\mathscr{G}}$ is $\varphi$-numerically trivial, then there exists a line bundle $\mathscr{L}$ on $Y$ such that $\operatorname{det}(\mathscr{G}) \cong \varphi^{*} \mathscr{L}$ (see [KMM87, Lemma 3.2.5]).

\section{Bott (partial) connection and applications}

In this section we provide technical tools for the proof of the main result.

3.1. (Bott connection) Let $X$ be a complex manifold, let $\mathscr{G} \subset T_{X}$ be a regular foliation, and set $\mathscr{N}=T_{X} / \mathscr{G}$. Let $p: T_{X} \rightarrow \mathscr{N}$ denotes the natural projection. For sections $U$ of $\mathscr{N}, T$ of $T_{X}$, and $V$ of $\mathscr{G}$ over some open subset of $X$ with $U=p(T)$, set $D_{V} U=p([V, U])$. This expression is well-defined, $\mathscr{O}_{X}$-linear in $V$ and satisfies the Leibnitz rule $D_{V}(f U)=f D_{V} U+(V f) U$ so that $D$ is a $\mathscr{G}$-connection on $\mathscr{N}$ (see [BB70]). We refer to it as the Bott connection on $\mathscr{N}$.

Lemma 3.2. Let $X$ be a complex manifold, and let $\mathscr{G} \subset T_{X}$ be a regular foliation with normal bundle $\mathscr{N}=T_{X} / \mathscr{G}$. Let $f: Z \rightarrow X$ be a compact manifold, and suppose that $f(Z)$ is tangent to $\mathscr{G}$. Then $f^{*} \mathscr{N}$ admits a flat holomorphic connection. In particular, Chern classes of $f^{*} \mathscr{N}$ vanish. 
Proof. One readily checks that the (partial) Bott connection on $\mathscr{N}$ gives a flat holomorphic connections on $f^{*} \mathscr{N}$.

3.3. (The Atiyah class of a locally free sheaf) Let $X$ be a complex manifold, and let $\mathscr{E}$ be a locally free sheaf on $X$. Let $J_{X}^{1}(\mathscr{E})$ be the sheaf of 1 -jets of $\mathscr{E}$. As a sheaf of abelian groups on $X$, $J_{X}^{1}(\mathscr{E}) \cong \mathscr{E} \oplus\left(\Omega_{X}^{1} \otimes \mathscr{E}\right)$, and the $\mathscr{O}_{X}$-module structure is given by $f(e, \alpha)=(f e, f \alpha-d f \otimes e)$, where $f, e$ and $\alpha$ are local sections of $\mathscr{O}_{X}, \mathscr{E}$ and $\Omega_{X}^{1} \otimes \mathscr{E}$, respectively. The Atiyah class of $\mathscr{E}$ (see [Ati57]) is defined to be the element at $(\mathscr{E}) \in H^{1}\left(X, \Omega_{X}^{1} \otimes \mathscr{E} n d_{\mathscr{O}_{X}}(\mathscr{E})\right)$ corresponding to the extension

$$
0 \rightarrow \Omega_{X}^{1} \otimes \mathscr{E} \rightarrow J_{X}^{1}(\mathscr{E}) \rightarrow \mathscr{E} \rightarrow 0
$$

The next result follows from the proof [BB70, Proposition 3.3] and [BB70, Corollary 3.4] (see also [AD14, Lemma 6.3]).

Lemma 3.4. Let $X$ be a complex manifold, and let $\mathscr{G} \subset T_{X}$ be a regular foliation. Set $\mathscr{N}:=T_{X} / \mathscr{G}$. Then at $(\mathscr{N}) \in H^{1}\left(X, \Omega_{X}^{1} \otimes \mathscr{E} n d_{\mathscr{O}_{X}}(\mathscr{N})\right)$ lies in the image of the natural map

$$
H^{1}\left(X, \mathscr{N}^{*} \otimes \mathscr{E}_{n} d_{\mathscr{O}_{X}}(\mathscr{N})\right) \rightarrow H^{1}\left(X, \Omega_{X}^{1} \otimes \mathscr{E}_{n} d_{\mathscr{O}_{X}}(\mathscr{N})\right)
$$

The first part of the statement of Lemma 3.5 below is [AD14, Lemma 6.4]. The proof of the second part of the statement is similar to that of [AD14, Lemma 6.4], and so we leave the details to the reader (see also [ST71, Theorem 1.14]). Notice that the statement of [AD14, Lemma 6.4] is slightly incorrect.

Lemma 3.5. Let $X$ be a complex projective manifold, and let $\mathscr{E}$ be a vector bundle on $X$. Let $U \subset X$ be an open set. Suppose that $X \backslash U$ has codimension $\geqslant m+2$ for some integer $m \geqslant 0$. Then

(1) $H^{i}(X, \mathscr{E}) \cong H^{i}(U, \mathscr{E} \mid U)$ for $0 \leqslant i \leqslant m$, and

(2) the natural map $H^{m+1}(X, \mathscr{E}) \rightarrow H^{m+1}(U, \mathscr{E} \mid U)$ is injective.

The following result generalizes [BB70, Corollary 3.4].

Lemma 3.6. Let $X$ be a complex projective manifold, and let $\mathscr{G} \subset T_{X}$ be a foliation of codimension q. Set $\mathscr{N}:=T_{X} / \mathscr{G}$. Suppose that the singular locus $Z(\mathscr{G})$ of $\mathscr{G}$ has codimension $\geqslant q+2$ in $X$. Then

(1) $c_{1}(\mathscr{N})^{q} \in H^{q}\left(X, \Omega_{X}^{q}\right)$ lies in the image of the natural map $H^{q}\left(X, \operatorname{det}(\mathscr{N})^{*}\right) \rightarrow H^{q}\left(X, \Omega_{X}^{q}\right)$, and

(2) $c_{1}(\mathscr{N})^{q+1} \equiv 0$.

Proof. Set $U:=X \backslash Z(\mathscr{G})$. By Lemma 3.4 and $\left[\right.$ Ati57, Theorem 6], $c_{1}\left(\mathscr{N}_{\mid U}\right) \in H^{1}\left(U, \Omega_{U}^{1}\right)$ lies in the image of the natural map

$$
H^{1}\left(U, \mathscr{N}_{\mid U}^{*}\right) \rightarrow H^{1}\left(U, \Omega_{U}^{1}\right) .
$$

This implies that $c_{1}\left(\mathscr{N}_{\mid U}\right)^{q} \in H^{q}\left(U, \Omega_{U}^{q}\right)$ lies in the image of the natural map

$$
H^{q}\left(U, \operatorname{det}\left(\mathscr{N}_{\mid U}\right)^{*}\right) \rightarrow H^{q}\left(U, \Omega_{U}^{q}\right)
$$

and that $c_{1}\left(\mathscr{N}_{\mid U}\right)^{q+1} \equiv 0$. On the other hand, by Lemma 3.5 above, the restriction map $\operatorname{det}\left(\mathscr{N}^{*}\right) \rightarrow$ $\operatorname{det}\left(\mathscr{N}_{\mid U}^{*}\right)$ induces an isomorphism $H^{q}\left(X, \operatorname{det}\left(\mathscr{N}^{*}\right)\right) \cong H^{q}\left(U, \operatorname{det}\left(\mathscr{N}_{\mid U}^{*}\right)\right)$ as well as an injective map $H^{q+1}\left(X, \operatorname{det}\left(\mathscr{N}^{*}\right)\right) \rightarrow H^{q+1}\left(U, \operatorname{det}\left(\mathscr{N}_{\mid U}^{*}\right)\right)$. The lemma then follows easily.

Lemma 3.8 below will prove to be crucial. Before starting with the proof, we recall the basic facts concerning Mori theory. 
3.7. (Fano-Mori contractions) Let $X$ be a complex projective manifold and consider the finite dimensional $\mathbb{R}$-vector space

$$
\mathrm{N}_{1}(X)=(\{1-\text { cycles }\} / \equiv) \otimes \mathbb{R}
$$

where $\equiv$ denotes numerical equivalence. The Mori cone of $X$ is the closure $\overline{\mathrm{NE}}(X) \subset \mathrm{N}_{1}(X)$ of the cone spanned by classes of effective curves. Every face $V$ of $\overline{\mathrm{NE}}(X)$ contained in $\left\{z \in \mathrm{N}_{1}(X) \mid K_{X} \cdot z<\right.$ $0\}$ corresponds to a surjective morphism with connected fibers $\varphi: X \rightarrow Y$ onto a normal projective variety, which is called a Fano-Mori contraction. The morphism $\varphi$ contracts precisely those curves on $X$ with class in $V$ (see [KMM87, Theorem 3.2.1]). Conversely, any morphism $\varphi: X \rightarrow Y$ with connected fibers onto a normal projective variety such that $-K_{X}$ is $\varphi$-ample arises in this way.

Lemma 3.8. Let $X$ be a complex projective manifold, and let $\mathscr{G} \subset T_{X}$ be a foliation of codimension $q$ on $X$. Suppose that $K_{\mathscr{G}} \equiv 0$ and that the singular locus $Z(\mathscr{G})$ of $\mathscr{G}$ has codimension $\geqslant q+2$. Let $\varphi: X \rightarrow Y$ be a Fano-Mori contraction, and let $F$ be an irreducible component of some fiber of $\varphi$. Then $\operatorname{dim} F \leq q$. If $\operatorname{dim} F>\operatorname{dim} X-\operatorname{dim} Y$ and $\operatorname{dim} F \geqslant \operatorname{dim} \varphi^{-1}(y)$ for all $y \in Y$, then we have $\operatorname{dim} F<q$.

Proof. Set $\mathscr{N}:=T_{X} / \mathscr{G}$. By Lemma 3.6, we have $\left(c_{1}(\operatorname{det}(\mathscr{N}))_{\mid F}\right)^{q+1} \equiv 0$. This implies that $\operatorname{dim} F \leqslant q$ since $c_{1}\left(\operatorname{det}(\mathscr{N})_{\mid F}\right) \equiv-K_{X \mid F}$ is ample by assumption.

Suppose from now on that $\operatorname{dim} F>\operatorname{dim} X-\operatorname{dim} Y$ and that $\operatorname{dim} F \geqslant \operatorname{dim} \varphi^{-1}(y)$ for any $y \in Y$. We argue by contradiction and assume that $\operatorname{dim} F=q$. Let $\nu: \widehat{F} \rightarrow F$ be a resolution of singularities. Consider the following commutative diagram:

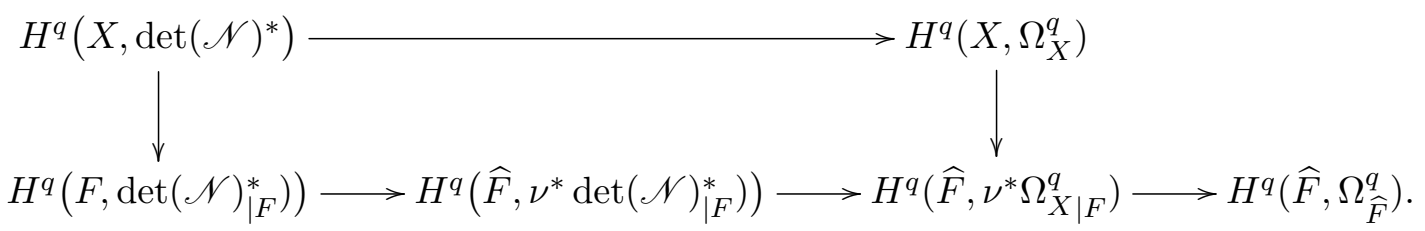

By Lemma 3.6 again, $c_{1}(X)^{q} \in H^{q}\left(X, \Omega_{X}^{q}\right)$ lies in the image of the map $H^{q}\left(X, \operatorname{det}(\mathscr{N})^{*}\right) \rightarrow$ $H^{q}\left(X, \Omega_{X}^{q}\right)$. Recall from [KMM87, Lemma 3.2.5] that there exists a line bundle $\mathscr{L}$ on $Y$ such that $\operatorname{det}(\mathscr{G}) \cong \varphi^{*} \mathscr{L}$, or equivalently $\operatorname{det}(\mathscr{N}) \cong \mathscr{O}_{X}\left(-K_{X}\right) \otimes \varphi^{*} \mathscr{L}^{*}$. By [AW97, Proposition 1.7], the group $\left.H^{q}\left(F, \mathscr{O}\left(K_{X}\right)_{\mid F}\right)\right)$ vanishes. It follows that the group $\left.H^{q}\left(F, \operatorname{det}(\mathscr{N})_{\mid F}^{*}\right)\right)$ vanishes as well. This implies that $\left(c_{1}(X)_{\mid}\right)^{q} \equiv 0$, yielding a contradiction as before. This completes the proof of the lemma.

\section{Projectable foliations under Fano-Mori contractions}

The proof of the main result relies on a criterion that guarantees that a given foliation is projectable under a morphism (see 2.7 for this notion), which we establish now.

Proposition 4.1. Let $X$ be a complex projective manifold, and let $\varphi: X \rightarrow Y$ be a Fano-Mori contraction with $\operatorname{dim} X-\operatorname{dim} Y \leqslant 2$. Let $\mathscr{G} \subset T_{X}$ be a foliation of positive rank $r$ such that $K_{\mathscr{G}}$ is $\varphi$-numerically trivial, and let $\tau_{Y} \in H^{0}\left(Y, \wedge^{r} T_{Y} \otimes \mathscr{L}^{*}\right)$ be the twisted field as in 2.7 (see Remark 2.8). Pick $y \in Y$ and set $F:=\varphi^{-1}(y)$. Suppose furthermore that $F \subset X \backslash Z(\mathscr{G})$ and that $\varphi$ is smooth along $F$. Then $y \notin Z\left(\tau_{Y}\right)$.

Before proving Proposition 4.1, we note the following immediate corollary. See Section 2.7 for the notion of projectable foliation.

Corollary 4.2. Let $X$ be a complex projective manifold, and let $\mathscr{G} \subset T_{X}$ be a foliation. Let $\varphi: X \rightarrow Y$ be a Fano-Mori contraction with $\operatorname{dim} X-\operatorname{dim} Y \leqslant 2$. Suppose that $K_{\mathscr{G}} \equiv 0$ and that $\operatorname{dim} Z(\mathscr{G}) \leqslant$ $\operatorname{dim} Y-1$. Then $\mathscr{G}$ is projectable under $\varphi$. 
Proof of Proposition 4.1. Set $m:=\operatorname{dim} Y$, and consider the following commutative diagram:

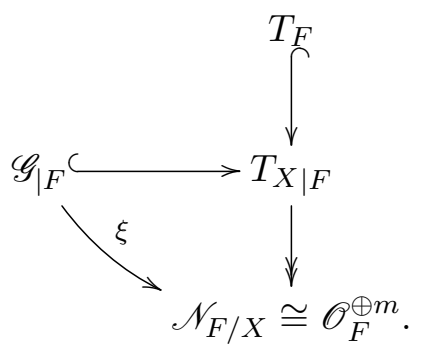

We argue by contradiction and assume that $\xi$ has generic rank $<r$. We denote by $\mathscr{K}$ its kernel, and by $\mathscr{Q}$ its image. Notice that $\mathscr{K}=\mathscr{G}_{\mid F} \cap T_{F} \subset T_{F}$ is a foliation on $F$. Since $\mathscr{Q} \subset \mathscr{N}_{F / X} \cong \mathscr{O}_{F}^{\oplus m}$, $\operatorname{det}(\mathscr{Q}) \cong \mathscr{O}_{F}(-D)$ for some effective divisor $D$ on $F$. By the adjunction formula, $-K_{F} \sim-K_{X \mid F}$ is ample. It follows that $\operatorname{det}\left(\mathscr{G}_{\mid F}\right) \cong \mathscr{O}_{F}$, and hence $\operatorname{det}(\mathscr{K}) \cong \mathscr{O}_{X}(D)$. Suppose first that $D \neq 0$. Applying [AD13, Proposition 7.5], we see that there exists a (rational) curve $C$ contained in $F$ and tangent to $\mathscr{K}$. But this contradicts Lemma 3.2 since $\operatorname{deg}\left(\operatorname{det}(\mathscr{N})_{\mid C}\right)=-K_{X} \cdot C>0$ where $\mathscr{N}:=T_{X} / \mathscr{G}$. Therefore, we must have $D=0$. Now either $\mathscr{K} \cong \mathscr{O}_{F}$, or $\mathscr{K}=T_{F}$ since $\operatorname{dim} F \leqslant 2$ by assumption. If $\mathscr{K} \cong \mathscr{O}_{F}$, then by Lemma 4.3 below, there exists a (rational) curve contained in $F$ and tangent to $\mathscr{G}$. If $\mathscr{K}=T_{F}$, then any rational curve contained in $F$ is tangent to $\mathscr{G}$. In either case, this contradicts Lemma 3.2 again, completing the proof of the proposition.

Lemma 4.3. Let $S$ be a smooth projective rational surface, and let $v$ be a nonzero holomorphic global vector field on $S$. Then there is a rational curve on $S$ tangent to $v$.

Proof. Denote by $S_{0}$ a minimal model of $S$, and let $v_{0}$ be the global holomorphic vector field induced by $v$ on $S_{0}$. Recall that either $S_{0} \cong \mathbb{P}^{2}$, or $S_{0}$ is a Hirzebruch surface. If $S_{0} \cong \mathbb{P}^{2}$, then it is well known that there is a line invariant by $v_{0}$ (see [Jou79, page 11]). Suppose that $S_{0}$ is a Hirzebruch surface. If $S \cong \mathbb{P}^{1} \times \mathbb{P}^{1}$, then there exist global vector fields $v_{1}$ and $v_{2}$ on $\mathbb{P}^{1}$ such that $v_{0}=p_{1}^{*} v_{1}+p_{2}^{*} v_{2}$, where $p_{1}$ and $p_{2}$ denote the projections on the two factors $\mathbb{P}^{1}$. Let $x_{1} \in \mathbb{P}^{1}$ such that $v_{1}\left(x_{1}\right)=0$. Then the curve $\left\{x_{1}\right\} \times \mathbb{P}^{1} \subset S$ is invariant by $v_{0}$. Finally, suppose that $S \neq \mathbb{P}^{1} \times \mathbb{P}^{1}$, and denote by $C \subset S$ the unique (rational) curve with negative self-intersection. The map

$$
\mathscr{O}_{C} \stackrel{v_{0 \mid C}}{\longrightarrow} T_{S \mid C} \rightarrow \mathscr{N}_{C / S}
$$

vanishes since $\operatorname{deg}\left(\mathscr{N}_{C / S}\right)=C \cdot C<0$, and thus $C$ is invariant by $v_{0}$.

Question 4.4. Let $X$ be a Fano manifold, and let $\mathscr{G}$ be a foliation of positive rank on $X$. Suppose that $K_{\mathscr{G}} \equiv 0$. Is it true that there exists a curve on $X$ tangent to $\mathscr{G}$ ?

Remark 4.5. If Question 4.4 has a positive answer, then Proposition 4.1 and Corollary 4.2 hold without restriction on $\operatorname{dim} X-\operatorname{dim} Y$. This is the case when $\mathscr{G}$ is regular by [Dru17, Theorem 1.2].

Proposition 4.6. Let $X$ be a complex projective manifold, and let $\mathscr{G} \subset T_{X}$ be a foliation of rank $r$ on $X$ with $K_{\mathscr{G}} \equiv 0$. Suppose that $X$ is the blow-up of a complex projective manifold $Y$ along a codimension 2 submanifold $B \subset Y$, and denote by $\varphi: X \rightarrow Y$ the natural morphism. Consider the projection $\mathscr{G}_{Y}$ of $\mathscr{G}$ on $Y$. Then $K_{\mathscr{G}_{Y}} \equiv 0$ and $Z\left(\mathscr{G}_{Y}\right)=\varphi(Z(\mathscr{G}))$. Moreover, if $B \not \subset \varphi(Z(\mathscr{G}))$, then $r \leqslant \operatorname{dim} X-2$ and $B \backslash Z\left(\mathscr{G}_{Y}\right)$ is invariant by $\mathscr{G}_{Y}$.

Proof. Let $\tau_{Y} \in H^{0}\left(Y, \wedge^{r} T_{Y} \otimes \mathscr{L}^{*}\right)$ be the twisted field as in 2.7 (see Remark 2.8).

Notice that $K_{\mathscr{G}_{Y}} \equiv c_{1}(\mathscr{L}) \equiv 0$ since $K_{\mathscr{G}} \equiv 0$. Thus, by 2.7 , we have $\varphi(Z(\mathscr{G})) \subset Z\left(\mathscr{G}_{Y}\right)=Z\left(\tau_{Y}\right)$. If $B \subset \varphi(Z(\mathscr{G}))$, then $Z\left(\mathscr{G}_{Y}\right)=\varphi(Z(\mathscr{G}))$ since $Z\left(\mathscr{G}_{Y}\right)$ and $\varphi(Z(\mathscr{G}))$ agree away from $B$.

Suppose from now on that $B \not \subset \varphi(Z(\mathscr{G}))$. Set $E:=\operatorname{Exc}(\varphi)$, and let $C \cong \mathbb{P}^{1}$ be a fiber of the natural map $E \rightarrow B$. Set also $y:=\varphi(C)$. Suppose that $C \subset X \backslash Z(\mathscr{G})$. By Lemma 3.2, $C$ is not tangent to 
$\mathscr{G}$. From 2.7, we conclude that $y \in Y \backslash Z\left(\mathscr{G}_{Y}\right)$. This shows that $Z\left(\mathscr{G}_{Y}\right) \cap B \subset \varphi(Z(\mathscr{G})) \cap B$, and thus $Z\left(\mathscr{G}_{Y}\right)=\varphi(Z(\mathscr{G}))$. Since $C$ is not tangent to $\mathscr{G}$, the inclusion $\mathscr{G}_{\mid C} \subset T_{X \mid C} \cong \mathscr{O}_{\mathbb{P}^{1}}(2) \oplus \mathscr{O}_{\mathbb{P}^{1}}^{\oplus \operatorname{dim} X-2} \oplus$ $\mathscr{O}_{\mathbb{P}^{1}}(-1)$ induces an inclusion $\mathscr{G}_{\mid C} \subset \mathscr{O}_{\mathbb{P}^{1}}^{\oplus \operatorname{dim} X-2} \oplus \mathscr{O}_{\mathbb{P}^{1}}(-1)$. This implies that $\mathscr{G}_{\mid C} \cong \mathscr{O}_{\mathbb{P}^{1}}^{\oplus r} \subset T_{E \mid C}$ since $\operatorname{det}\left(\mathscr{G}_{\mid C}\right) \cong \mathscr{O}_{C}$. It follows that $E$ is invariant by $\mathscr{G}$, and that $r \leqslant \operatorname{dim} X-2$. The proposition follows easily.

In Lemma 4.7 below, we gather some properties of conic bundles for later reference. Recall that a conic bundle structure on a complex projective manifold $X$ is a surjective morphism $\varphi: X \rightarrow Y$ onto a complex projective manifold with fibers isomorphic to conics. Its discriminant locus is the set $\Delta:=\left\{y \in Y \mid\right.$ such that $X_{y}:=\varphi^{-1}(y)$ is not smooth $\}$.

Lemma 4.7. Let $X$ be a complex projective manifold, and let $\varphi: X \rightarrow Y$ be a conic bundle structure on $X$. Suppose that its discriminant locus $\Delta$ is non-empty. Set $\Delta_{1}:=\left\{y \in \Delta \mid X_{y}\right.$ is reduced $\}$ and $\Delta_{2}:=\left\{y \in \Delta \mid X_{y}\right.$ is not reduced $\}$. Then $\Delta$ is an hypersurface in $Y$, and $\Delta_{1} \subset \Delta$ is a dense open set. Moreover, $\Delta$ has normal crossing singularities in codimension $1, \Delta_{1} \subset \Delta_{\mathrm{reg}}$, and $\Delta$ is singular along codimension 1 irreducible components of $\Delta_{2}$. Further, if $y$ is any point on $Y$ and $F:=\varphi^{-1}(y)$, then the following properties hold in addition.

(1) If $y \in \Delta_{1}$, then $F=C_{1} \cup C_{2}$ is the union of 2 smooth rational curves meeting transversally at a point, $-K_{X} \cdot C_{i}=1$ and $\mathscr{N}_{C_{i} / X} \cong \mathscr{O}_{\mathbb{P}^{1}}^{\oplus \operatorname{dim} X-2} \oplus \mathscr{O}_{\mathbb{P}^{1}}(-1)$ for $i \in\{1,2\}$.

(2) If $y \in \Delta_{2} \backslash \Delta_{\text {reg, }}$, then $F$ is a smooth rational curve, $-K_{X} \cdot F=1$, and $\mathscr{N}_{F / X} \cong \mathscr{O}_{\mathbb{P 1}}^{\oplus \operatorname{dim} X-3} \oplus$ $\mathscr{O}_{\mathbb{P}^{1}}(1) \oplus \mathscr{O}_{\mathbb{P}^{1}}(-2)$.

(3) Let $\widehat{\Delta}$ denotes the Zariski closure in $X$ of the singular locus of $\varphi^{-1}\left(\Delta_{1}\right)$. There exists an open set $\Delta^{\circ} \subset \Delta$ with complement of codimension $\geqslant 2$ in $\Delta$ such that the natural map $\widehat{\Delta} \cap \varphi^{-1}\left(\Delta^{\circ}\right) \rightarrow \Delta^{\circ}$ identifies with the normalization morphism.

Proof. The same argument used in the proof of [Bea77, Proposition 1.2] shows that $\Delta$ has pure codimension 1 , and that $\Delta_{2}$ has codimension $\geqslant 1$ in $\Delta$. It also shows that $\Delta_{1}$ is smooth, that $\Delta$ has normal crossing singularities in codimension 1 , and that $\Delta$ is singular along codimension 1 irreducible components of $\Delta_{2}$.

Statements (1) and (2) are due to Ando (see [And85]). Statement (3) follows easily from [Bea77, Lemme 1.5.2].

Proposition 4.8. Let $X$ be a complex projective manifold, and let $\mathscr{G} \subset T_{X}$ be a foliation of rank $r$ on $X$. Suppose that $K_{\mathscr{G}} \equiv 0$ and that $\operatorname{dim} Z(\mathscr{G}) \leqslant \operatorname{dim} X-3$. Let $\varphi: X \rightarrow Y$ be a conic bundle structure on $X$ with discriminant locus $\Delta$. Then $\mathscr{G}$ is projectable under $\varphi$ by Corollary 4.2. Consider the projection $\mathscr{G}_{Y}$ of $\mathscr{G}$ on $Y$. Then $K_{\mathscr{G}_{Y}} \equiv 0$ and $\operatorname{dim} Z\left(\mathscr{G}_{Y}\right) \leqslant \max (\operatorname{dim} Z(\mathscr{G}), \operatorname{dim} Y-3)$. Moreover, if $\Delta$ is non-empty, then $r \leqslant \operatorname{dim} X-2$ and $\Delta \backslash Z\left(\mathscr{G}_{Y}\right)$ is invariant by $\mathscr{G}_{Y}$.

Proof. Let $\tau_{Y} \in H^{0}\left(Y, \wedge^{r} T_{Y} \otimes \mathscr{L}^{*}\right)$ be the twisted field as in 2.7 (see Remark 2.8). By Proposition 4.1, $Z\left(\tau_{Y}\right) \backslash \Delta=\varphi(Z(\mathscr{G})) \backslash \Delta$.

If $\Delta=\emptyset$, then $Z\left(\tau_{Y}\right)$ has codimension at least 2 in $Y$, and thus $Z\left(\mathscr{G}_{Y}\right)=Z\left(\tau_{Y}\right)$ and $K_{\mathscr{G}_{Y}} \equiv$ $-c_{1}(\mathscr{L}) \equiv 0$. Moreover, $\operatorname{dim} Z\left(\mathscr{G}_{Y}\right) \leqslant \operatorname{dim} Z(\mathscr{G})$.

Suppose from now on that $\Delta \neq \emptyset$. Set $\Delta_{1}:=\left\{y \in \Delta \mid X_{y}\right.$ is reduced $\}$ and $\Delta_{2}:=\{y \in$ $\Delta \mid X_{y}$ is not reduced $\}$.

Let $y \in \Delta_{1} \backslash \varphi(Z(\mathscr{G}))$, and set $F:=\varphi^{-1}(y)$. Then $F=C_{1} \cup C_{2}$ is the union of 2 smooth rational curves meeting transversally at a point, and $-K_{X} \cdot C_{i}=1$. By Lemma 3.2, the curve $C_{i}$ is not tangent to $\mathscr{G}$, and thus $y \in Y \backslash Z\left(\tau_{Y}\right)$. This immediately implies that $Z\left(\tau_{Y}\right) \backslash \Delta_{2}=\varphi(Z(\mathscr{G})) \backslash \Delta_{2}$. As before, we conclude that $Z\left(\mathscr{G}_{Y}\right)=Z\left(\tau_{Y}\right)$ and that $K_{\mathscr{G}_{Y}} \equiv-c_{1}(\mathscr{L}) \equiv 0$. Since $C_{i}$ is not tangent to $\mathscr{G}$, the inclusion $\mathscr{G}_{\mid C_{i}} \subset T_{X \mid C_{i}}$ induces an inclusion $\mathscr{G}_{\mid C_{i}} \subset \mathscr{N}_{C_{i} / X} \cong \mathscr{O}_{\mathbb{P}^{1}}^{\oplus \operatorname{dim} X-2} \oplus \mathscr{O}_{\mathbb{P}^{1}}(-1)$. It follows 
that $\mathscr{G}_{\mid C_{i}} \cong \mathscr{O}_{\mathbb{P}}^{\oplus r}$ since $\operatorname{det}\left(\mathscr{G}_{\mid C_{i}}\right) \cong \mathscr{O}_{C_{i}}$. This in turn implies that $\varphi^{-1}(\Delta)$ is invariant by $\mathscr{G}$, and that $\Delta \backslash Z\left(\mathscr{G}_{Y}\right)$ is invariant by $\mathscr{G}_{Y}$. Moreover, we have $r \leqslant \operatorname{dim} X-2$.

Now, we proceed to show that $\operatorname{dim} Z\left(\mathscr{G}_{Y}\right) \leqslant \max (\operatorname{dim} Z(\mathscr{G}), \operatorname{dim} Y-3)$. Let $\widehat{\Delta}$ denotes the Zariski closure in $X$ of the singular locus of $\varphi^{-1}\left(\Delta_{1}\right)$. By Lemma 4.7, there exists an open set $\Delta^{\circ} \subset \Delta$ with complement of codimension $\geqslant 2$ in $\Delta$ such that the natural map $\widehat{\Delta} \cap \varphi^{-1}\left(\Delta^{\circ}\right) \rightarrow \Delta^{\circ}$ identifies with the normalization morphism. By shrinking $\Delta^{\circ}$ if necessary, we may assume without loss of generality that $\Delta^{\circ}$ has normal crossing singularities. This implies that $\widehat{\Delta} \cap \varphi^{-1}\left(\Delta^{\circ}\right)$ is smooth, and that the restriction of $d_{x} \varphi$ to $T_{x} \widehat{\Delta}$ is injective. Since $\hat{\Delta}$ is a union of irreducible components of the singular locus of $\varphi^{-1}(\Delta)$ and since $\varphi^{-1}(\Delta)$ is invariant by $\mathscr{G}$, we conclude that $\widehat{\Delta} \backslash Z(\mathscr{G})$ is invariant by $\mathscr{G}$ as well. This shows that $Z\left(\mathscr{G}_{Y}\right) \cap \Delta^{\circ}=\varphi(Z(\mathscr{G})) \cap \Delta^{\circ}$, and hence $\operatorname{dim} Z\left(\mathscr{G}_{Y}\right) \leqslant \max (\operatorname{dim} Z(\mathscr{G}), \operatorname{dim} Y-3)$ since $\Delta \backslash \Delta^{\circ}$ has codimension $\geqslant 3$ in $X$. This finishes the proof of the proposition.

Proposition 4.9. Let $X$ be a complex projective manifold, and let $\mathscr{G} \subset T_{X}$ be a foliation of rank $r$ on $X$. Suppose that $K_{\mathscr{G}} \equiv 0$ and that $\operatorname{dim} Z(\mathscr{G}) \leqslant r-2$. Let $\varphi: X \rightarrow Y$ be a Fano-Mori contraction with $\operatorname{dim} Y=r$. Suppose furthermore that $\mathscr{G}$ is projectable under $\varphi$. Then $Z(\mathscr{G})=\emptyset, \varphi$ is a smooth morphism, and $\mathscr{G}$ yields a flat holomorphic connection on $\varphi$. In particular, we have $K_{Y} \equiv 0$.

Proof. Set $\mathscr{N}:=T_{X} / \mathscr{G}$. By [KMM87, Lemma 3.2.5], there exists a line bundle $\mathscr{L}$ on $Y$ such that $\operatorname{det}(\mathscr{G}) \cong \varphi^{*} \mathscr{L}$. Notice that $c_{1}(\mathscr{L}) \equiv 0$ since $K_{\mathscr{G}} \equiv 0$.

We first show that $\mathscr{L} \cong \mathscr{O}_{Y}\left(-K_{Y}\right)$. Set $Y^{\circ}:=Y_{\text {reg }} \backslash \varphi(Z(\mathscr{G}))$, and notice that $\operatorname{codim} Y \backslash Y^{\circ} \geqslant 2$. Set $X^{\circ}:=\varphi^{-1}\left(Y^{\circ}\right)$. Let $y \in Y^{\circ}$, and let $F$ be an irreducible component of $\varphi^{-1}(y)$. Denote by $\nu: \widehat{F} \rightarrow F$ a resolution of singularities. Notice that $\operatorname{dim} F=\operatorname{dim} X-\operatorname{dim} Y=: q$ by Lemma 3.8 since $\operatorname{dim} Y=r$.

By Lemma 3.4, the Atiyah class at $\left(\mathscr{N}_{\mid X^{\circ}}\right) \in H^{1}\left(X^{\circ}, \Omega_{X^{\circ}}^{1} \otimes \mathscr{E} n d_{\mathscr{O}_{X^{\circ}}}\left(\mathscr{N}_{\mid X^{\circ}}\right)\right)$ lies in the image of the natural map

$$
H^{1}\left(X^{\circ}, \mathscr{N}_{\mid X^{\circ}}^{*} \otimes \mathscr{E}_{n} d_{\mathscr{O}_{X^{\circ}}}\left(\mathscr{N}_{\mid X^{\circ}}\right)\right) \rightarrow H^{1}\left(X^{\circ}, \Omega_{X^{\circ}}^{1} \otimes \mathscr{E}_{n} d_{\mathscr{O}_{X^{\circ}}}\left(\mathscr{N}_{\mid X^{\circ}}\right)\right) .
$$

In particular, there exists $\alpha \in H^{1}\left(X^{\circ}, \mathscr{N}_{X^{\circ}}^{*}\right)$ mapping to $c_{1}\left(\mathscr{N}_{\mid X^{\circ}}\right) \in H^{1}\left(X^{\circ}, \Omega_{X^{\circ}}^{1}\right)$. Notice that $\alpha^{q} \in H^{q}\left(X^{\circ}, \operatorname{det}\left(\mathscr{N}_{\mid X^{\circ}}^{*}\right)\right) \cong H^{q}\left(X^{\circ}, \mathscr{O}_{X^{\circ}}\left(K_{X^{\circ}}\right) \otimes \varphi_{\mid X \circ}^{*} \mathscr{L}_{\mid Y^{\circ}}\right)$ maps to $c_{1}\left(\mathscr{N}_{\mid X^{\circ}}\right)^{q} \in H^{q}\left(X^{\circ}, \Omega_{X^{\circ}}^{q}\right)$. Let $\beta \in H^{0}\left(Y^{\circ}, R^{q} \varphi_{\mid X^{\circ}{ }^{\circ}} \mathscr{O}_{X^{\circ}}\left(K_{X^{\circ}}\right) \otimes \mathscr{L}_{\mid Y^{\circ}}\right)$ denotes the image of $\alpha^{q}$ under the edge map

$$
H^{q}\left(X^{\circ}, \mathscr{O}_{X^{\circ}}\left(K_{X^{\circ}}\right) \otimes \varphi_{\mid X \circ}^{*} \mathscr{L}_{\mid Y^{\circ}}\right) \rightarrow H^{0}\left(Y^{\circ}, R^{q} \varphi_{\mid X^{\circ}}{ }_{*} \mathscr{O}_{X^{\circ}}\left(K_{X^{\circ}}\right) \otimes \mathscr{L}_{\mid Y^{\circ}}\right) .
$$

Now, consider the following commutative diagram:

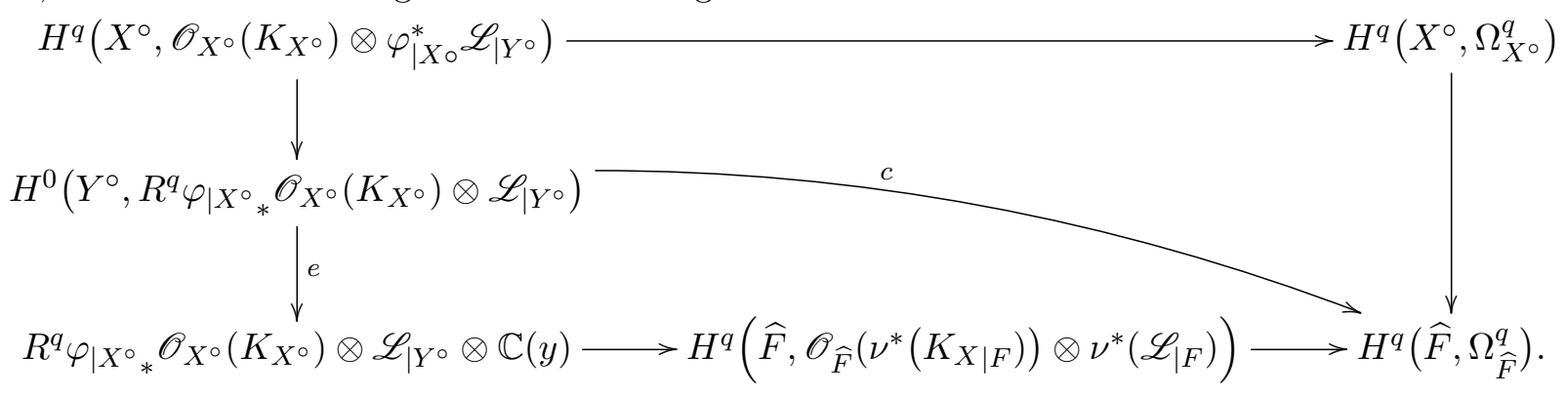

Then

$$
c(\beta)=(-1)^{q} \cdot\left(\nu^{*}\left(c_{1}(X)_{\mid F}\right)\right)^{q} \in H^{q}\left(\widehat{F}, \Omega_{\widehat{F}}^{q}\right) .
$$

Since $-K_{X}$ is $\varphi$-ample and $\operatorname{dim} \widehat{F}=q$, we have $\left(\nu^{*}\left(c_{1}(X)_{\mid F}\right)\right)^{q} \neq 0$, and hence $e(\beta) \neq 0$. On the other hand, by [Kol86, Proposition 7.6], we have $R^{q} \varphi_{X^{\circ}{ }_{*}} \mathscr{O}_{X^{\circ}}\left(K_{X^{\circ}}\right) \cong \mathscr{O}_{Y^{\circ}}\left(K_{Y^{\circ}}\right)$, and thus $\beta$ is a nowhere vanishing section of $\mathscr{O}_{Y^{\circ}}\left(K_{Y^{\circ}}\right) \otimes \mathscr{L}_{\mid Y^{\circ}}$. This implies that $\mathscr{L} \cong \mathscr{O}_{Y}\left(-K_{Y}\right)$ since $\operatorname{codim} Y \backslash Y^{\circ} \geqslant 2$, proving our claim. 
Since $\mathscr{G}$ is projectable under $\varphi$, the natural map $T_{X / Y} \oplus \mathscr{G} \rightarrow T_{X}$ is generically injective. A straightforward local computation shows that $\operatorname{det}\left(T_{X / Y}\right) \cong \mathscr{O}_{X}\left(-K_{X / Y}+R(\varphi)\right)$, where $R(\varphi)$ denotes the ramification divisor of $\varphi$, and thus

$$
\operatorname{det}\left(T_{X / Y} \oplus \mathscr{G}\right) \cong \operatorname{det}\left(T_{X / Y}\right) \otimes \operatorname{det}(\mathscr{G}) \cong \mathscr{O}_{X}\left(-K_{X}+R(\varphi)\right) .
$$

This implies that $R(\varphi)=0$, and that $T_{X / Y} \oplus \mathscr{G} \cong T_{X}$. It follows that $T_{X / Y}$ and that $\mathscr{G}$ are locally free sheaves on $X$ and that $Z(\mathscr{G})=\emptyset$. From [Dru17, Lemma 4.1], we conclude that $\varphi$ is a smooth morphism. Moreover, $\mathscr{G}$ induces a flat (holomorphic) connection on $\varphi$, completing the proof of the proposition.

\section{Codimension 2 (regular) foliations with numerically trivial canonical class}

In this section we prove Theorem 1.1. Notice that Theorem 1.1 is an immediate consequence of Theorem 5.2 below.

The statement of Proposition 5.1 is contained in [LPT11a, Corollary 4.7]. We first give a new proof of this result.

Proposition 5.1. Let $X$ be a complex projective manifold, and let $\mathscr{G} \subset T_{X}$ be a foliation of codimension 1 on $X$ with $K_{\mathscr{G}} \equiv 0$. Suppose that $\operatorname{dim} Z(\mathscr{G}) \leqslant \operatorname{dim} X-3$. Suppose furthermore that $K_{X}$ is not nef. Then $Z(\mathscr{G})=\emptyset$.

Proof. Let $\varphi: X \rightarrow Y$ be the contraction of a $K_{X}$-negative extremal ray $R \subset \overline{\mathrm{NE}}(X)$. By Lemma 3.8, $\operatorname{dim} Y=\operatorname{dim} X-1$ and $\varphi$ is equidimensional. Applying [Wiś91, Theorem 1.2], we see that $\varphi$ is a conic bundle. Applying Corollary 4.2, we see that $\mathscr{G}$ is projectable under $\varphi$. The proposition then follows from Proposition 4.9.

Theorem 5.2. Let $X$ be a complex projective manifold, and let $\mathscr{G} \subset T_{X}$ be a foliation of codimension 2 on $X$ with $K_{\mathscr{G}} \equiv 0$. Suppose that $\operatorname{dim} Z(\mathscr{G}) \leqslant \operatorname{dim} X-4$. Suppose furthermore that $K_{X}$ is not nef. Then $Z(\mathscr{G})=\emptyset$ and one of the following holds.

(1) There exist a complex projective manifold $Y$, a regular codimension 2 foliation $\mathscr{G}_{Y}$ on $Y$ with $K_{\mathscr{G}_{Y}} \equiv 0$, and a compact leaf $Z \subset Y$ of $\mathscr{G}_{Y}$ such that $X$ is the blow-up of $Y$ along $Z$. Moreover, $\mathscr{G}$ is the pull-back of $\mathscr{G}_{Y}$.

(2) There exists a conic bundle structure $\varphi: X \rightarrow Y$ on $X$ onto a complex projective manifold $Y$, $\mathscr{G}$ is generically transverse to $\varphi$ and induces a regular codimension 1 foliation $\mathscr{G}_{Y}$ on $Y$ with $K_{\mathscr{G}_{Y}} \equiv 0$. Moreover, the discriminant locus of $\varphi$ is either empty or a union of leaves of $\mathscr{G}_{Y}$.

(3) There exists a smooth morphism $\varphi: X \rightarrow Y$ onto a complex projective manifold $Y$ of dimension $\operatorname{dim} Y=\operatorname{dim} X-2$ with $K_{Y} \equiv 0$, and $\mathscr{G}$ yields a flat holomorphic connection on $\varphi$.

Proof. Let $\varphi: X \rightarrow Y$ be the contraction of a $K_{X}$-negative extremal ray $R \subset \overline{\mathrm{NE}}(X)$, and let $F$ be an irreducible component of some fiber of $\varphi$. Applying Lemma 3.8 to $\varphi$, we see that one of the following holds:

(1) $\operatorname{dim} Y=\operatorname{dim} X-2$ and $\varphi$ is equidimensional,

(2) $\operatorname{dim} Y=\operatorname{dim} X-1$ and $\varphi$ is equidimensional,

(3) $\operatorname{dim} Y=\operatorname{dim} X$ and $\operatorname{dim} F \leqslant 1$. 
By Corollary $4.2, \mathscr{G}$ is projectable under $\varphi$.

If we are in case (1), then the theorem follows from Proposition 4.9.

Suppose that we are in case (2). Applying [Wiś91, Theorem 1.2], we see that $\varphi$ is a conic bundle. By Proposition 4.8, we have $K \mathscr{G}_{Y} \equiv 0$ and $\operatorname{dim} Z\left(\mathscr{G}_{Y}\right) \leqslant \operatorname{dim} Y-3$. By [LPT11b, Theorem 5.4] and Proposition 5.1 applied to $\mathscr{G}_{Y}$, the singular set $Z\left(\mathscr{G}_{Y}\right)$ is empty. This implies that $Z(\mathscr{G})=\emptyset$ as well (see 2.7). Apply again Proposition 4.8 to conclude that the discriminant locus $\Delta$ of $\varphi$ is either empty or a union of leaves of $\mathscr{G}_{Y}$.

Finally, suppose that we are in case (3). By [Wiś91, Theorem 1.2], $Y$ is smooth and $\varphi$ is the blow-up of $Y$ along a codimension 2 submanifold $Z \subset Y$. By Proposition 4.6, $K \mathscr{G}_{Y} \equiv 0, \operatorname{dim} Z\left(\mathscr{G}_{Y}\right) \leqslant$ $\operatorname{dim} Z(\mathscr{G}) \leqslant \operatorname{dim} Y-4$, and $Z(\mathscr{G})$ is empty if and only if so is $Z\left(\mathscr{G}_{Y}\right)$.

Suppose that $Z(\mathscr{G})$ is non-empty. In particular, $Z\left(\mathscr{G}_{Y}\right)$ is also non-empty. If moreover $K_{Y}$ is not nef, then there exists an elementary Fano-Mori contraction on $Y$ as in case (3). This yields a finite sequence of contractions

$$
Y_{0}:=X \rightarrow Y_{1}:=Y \rightarrow Y_{2} \rightarrow \cdots \rightarrow Y_{i} \rightarrow Y_{i+1} \rightarrow \cdots \rightarrow Y_{m}
$$

where $Y_{i}$ is a complex projective manifold, $\varphi_{i}: Y_{i} \rightarrow Y_{i+1}$ is the blow-up of a codimension 2 submanifold $Z_{i+1} \subset Y_{i+1}$, and $K_{Y_{m}}$ is nef. Note that the process ends since $\rho\left(Y_{i+1}\right)<\rho\left(Y_{i}\right)$. Moreover, the projection $\mathscr{G}_{i}$ of $\mathscr{G}_{\text {on }} Y_{i}$ is a codimension 2 foliation with $K \mathscr{G}_{i} \equiv 0$ and non-empty singular set $Z\left(\mathscr{G}_{i}\right)$. This contradicts [LPT11b, Theorem 5.4] applied to $\mathscr{G}_{m}$, proving that $Z(\mathscr{G})=\emptyset$. Applying Proposition 4.6 again, we see that $Z\left(\mathscr{G}_{Y}\right)=\emptyset$, and that $Z$ is a leaf of $\mathscr{G}_{Y}$. This completes the proof of the theorem.

\section{Algebraically integrable regular foliations with numerically trivial canonical class}

In this section we prove Theorem 1.2. The proof relies on a global version of Reeb stability theorem (see Proposition 6.6), which we establish first.

The following notation is used in the formulation of Proposition 6.6.

Definition 6.1. Let $X$ be a normal projective variety, let $H$ be an ample Cartier divisor on $X$, and let $\mathscr{G}$ be a reflexive coherent sheaf of $\mathscr{O}_{X}$-modules. We say that $\mathscr{G}$ is strongly stable with respect to $H$ if, for any normal projective variety $Z$ and any generically finite surjective morphism $f: Z \rightarrow X$, the reflexive pull-back sheaf $\left(f^{*} \mathscr{G}\right)^{* *}$ is $f^{*} H$-stable.

6.2. (The holonomy group of a stable reflexive sheaf) Let $X$ be a normal complex projective variety, and let $\mathscr{G}$ be a reflexive sheaf on $X$. Suppose that $\mathscr{G}$ is stable with respect to an ample Cartier divisor $H$ and that $\mu_{H}(\mathscr{G})=0$. For a sufficiently large positive integer $m$, let $C \subset X$ be a general complete intersection curve of elements in $|m H|$. Let $x \in C$. By the restriction theorem of Mehta and Ramanathan, the locally free sheaf $\mathscr{G}_{\mid C}$ is stable with $\operatorname{deg}\left(\mathscr{G}_{\mid C}\right)=0$, and hence it corresponds to a unique unitary representation $\rho: \pi_{1}(C, x) \rightarrow \mathbb{U}\left(\mathscr{G}_{x}\right)$ by a result of Narasimhan and Seshadri ([NS65]). The holonomy group $\operatorname{Hol}_{x}(\mathscr{G})$ of $\mathscr{G}$ is the Zariski closure of $\rho\left(\pi_{1}(C, x)\right)$ in $\mathrm{GL}\left(\mathscr{G}_{x}\right)$. It does not depend on $C \ni x$ provided that $m$ is large enough (see [BK08]).

We will need the following observation.

Lemma 6.3. Let $X$ be a normal complex projective variety, let $x$ be a general point on $X$, and let $\mathscr{G}$ be a coherent sheaf of $\mathscr{O}_{X}$-modules. Suppose that $\mathscr{G}$ is stable with respect to an ample divisor $H$ and that $\mu_{H}(\mathscr{G})=0$. Suppose furthermore that its holonomy group $\operatorname{Hol}_{x}(\mathscr{G})$ is connected. Then $\mathscr{G}$ is strongly stable with respect to $H$. 
Proof. Let $Z$ be a normal projective variety, and let $g: Z \rightarrow X$ be a generically finite surjective morphism. The map $g$ factorizes into $Z \rightarrow Y \rightarrow X$ where $Y$ is a normal projective variety, $b: Z \rightarrow Y$ is a birational map, and $f: Y \rightarrow X$ is a finite cover. The same argument used in the proof of [Dru16, Lemma 6.22] shows that the reflexive pull-back sheaf $\left(f^{*} \mathscr{G}\right)^{* *}$ is $f^{*} H$-stable. One only needs to replace the use of [Kem92, Theorem 1] with [HL97, Lemma 3.2.3].

We argue by contradiction and assume that $\left(g^{*} \mathscr{G}\right)^{* *}$ is not $g^{*} H$-stable. It follows that there exists $\mathscr{E} \subset\left(g^{*} \mathscr{G}\right)^{* *}$ with $0<\operatorname{rank} \mathscr{E}<\operatorname{rank}\left(g^{*} \mathscr{G}\right)^{* *}$ and $\mu_{g^{*} H}(\mathscr{E}) \geqslant \mu_{g^{*} H}\left(\left(g^{*} \mathscr{G}\right)^{* *}\right)=\mu_{H}(\mathscr{G})=0$. Then $b_{*} \mathscr{E} \subset\left(f^{*} \mathscr{G}\right)^{* *}$ since $b_{*}\left(\left(g^{*} \mathscr{G}\right)^{* *}\right)$ and $\left(f^{*} \mathscr{G}\right)^{* *}$ agree over an open subset with complement of codimension at least two. On the other hand, $\mu_{f^{*} H}\left(b_{*} \mathscr{E}\right)=\mu_{g^{*} H}(\mathscr{E})=0$. This yields a contradiction, finishing the proof of the lemma.

The following result is probably well-known to experts. We include a proof here for the reader's convenience.

Lemma 6.4. Let $X$ be a complex projective manifold, and $\varphi: X \rightarrow Y$ be a smooth morphism onto a complex projective manifold. Suppose that $K_{X}$ is pseudo-effective, and suppose that $K_{X / Y} \equiv 0$. Then there exist complex projective manifolds $B$ and $F$ as well as a finite étale cover $f: B \times F \rightarrow X$ such that $T_{B \times F / B}=f^{*} T_{X / Y}$.

Proof. By [LPT11b, Theorem 5.2], the divisor $K_{X / Y}$ is a torsion point, and hence, by replacing $X$ with a finite étale cover, if necessary, we may assume without loss of generality that $K_{X / Y} \sim 0$. Applying [LPT11b, Theorem 5.2] again, we see that $\varphi$ admits a holomorphic connection. In particular, $\varphi$ is a locally trivial fibration for the Euclidean topology. Let $F$ be any fiber of $\varphi$. By the adjunction formula, $K_{F} \sim 0$. Let $y \in Y$, and denote by $X_{y} \cong F$ the fiber $\varphi^{-1}(y)$. Let $\operatorname{Aut}^{\circ}\left(X_{y}\right)$ denotes the neutral component of the automorphism group $\operatorname{Aut}\left(X_{y}\right)$ of $X_{y}$. Then $\operatorname{Aut}^{\circ}\left(X_{y}\right)$ is an abelian variety of dimension $h^{0}\left(F, T_{F}\right)$. Recall from [GP11, Exposé $\mathrm{VI}_{\mathrm{B}}$, Théorème 3.10] that the algebraic groups $\operatorname{Aut}^{\circ}\left(X_{y}\right)$ fit together to form an abelian scheme $\mathscr{A}$ over $Y$. Since $\mathscr{A}$ is locally trivial, there exist an abelian variety $A$, and a finite étale cover $Y_{1} \rightarrow Y$ such that $\mathscr{A} \times_{Y} Y_{1} \cong A \times Y_{1}$ as group schemes over $Y_{1}$. This follows from the fact that there is a fine moduli scheme for polarized abelian varieties of dimension $g$, with level $N$ structure and polarization of degree $d$ provided that $N$ is large enough. In particular, $A$ acts faithfully on $X_{1}:=X \times_{Y} Y_{1}$. By [Bri10, Proof of Theorem 1.2, page 10], there exist a finite étale cover $X_{2}$ of $X_{1}$ equipped with a faithful action of $A$, and an $A$-isomorphism $X_{2} \cong A \times Z_{2}$ for some projective manifold $Z_{2}$, where $A$ acts trivially on $Z_{2}$ and diagonally on $A \times Z_{2}$. One readily checks that there exists a smooth morphism with connected fibers $\varphi_{2}: Z_{2} \rightarrow Y_{2}$ as well as a finite étale cover $Y_{2} \rightarrow Y_{1}$ and a commutative diagram:

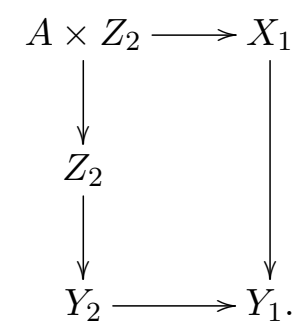

This implies that $K_{A \times Z_{2} / Y_{2}} \sim 0$, and thus we also have $K_{Z_{2} / Y_{2}} \sim 0$. Repeating the process finitely many times, if necessary, we may assume without loss of generality that any fiber $F_{2}$ of $\varphi_{2}$ satisfies $h^{0}\left(F_{2}, T_{F_{2}}\right)=0$. Notice that $K_{F_{2}} \sim 0$ by the adjunction formula. Moreover,

$$
\begin{aligned}
h^{1}\left(F_{2}, \mathscr{O}_{F_{2}}\right) & =h^{\operatorname{dim} F_{2}-1}\left(F_{2}, \mathscr{O}_{F_{2}}\right) & & \text { by Serre duality } \\
& =h^{0}\left(F_{2}, \Omega_{F_{2}}^{\operatorname{dim} F_{2}-1}\right) & & \text { by Hodge symmetry } \\
& =h^{0}\left(F_{2}, T_{F_{2}}\right) & & \text { for } \Omega_{F_{2}}^{\operatorname{dim} F_{2}-1} \cong T_{F_{2}} \\
& =0 . & &
\end{aligned}
$$


Let $H_{2}$ be an ample divisor on $Z_{2}$. Since $\varphi_{2}$ admits a flat holomorphic connection, it is given by a representation

$$
\pi_{1}\left(Y_{2}, y\right) \rightarrow \operatorname{Aut}\left(F_{2}, H_{2 \mid F_{2}}\right)
$$

where $y:=\varphi_{2}\left(F_{2}\right)$, and where $\operatorname{Aut}\left(F_{2}, H_{2 \mid F_{2}}\right)$ denotes the group $\left\{u \in \operatorname{Aut}\left(F_{2}\right) \mid u^{*} H_{2 \mid F_{2}} \equiv H_{2 \mid F_{2}}\right\}$. Since $h^{1}\left(F_{2}, \mathscr{O}_{F_{2}}\right)=0$, the Picard group of $F_{2}$ is discrete. The subgroup $\operatorname{Pic}^{\tau}\left(F_{2}\right) \subset \operatorname{Pic}\left(F_{2}\right)$ of invertible sheaves with numerically trivial first Chern class is therefore finite. By replacing $\mathrm{H}_{2}$ with $\mathrm{mH}_{2}$ for some positive integer $m$, if necessary, we may assume without loss of generality, that $\operatorname{Aut}\left(F_{2}, H_{2 \mid F_{2}}\right)=\left\{u \in \operatorname{Aut}\left(F_{2}\right) \mid u^{*} H_{2 \mid F_{2}} \sim H_{2 \mid F_{2}}\right\}$. This implies that $\operatorname{Aut}\left(F_{2}, H_{2 \mid F_{2}}\right)$ is an affine algebraic group. It follows that $\operatorname{Aut}\left(F_{2}, H_{2 \mid F_{2}}\right)$ is finite since since $h^{0}\left(F_{2}, T_{F_{2}}\right)=0$. Therefore, replacing $Y_{2}$ with a finite étale cover, if necessary, we may assume that $Z_{2} \cong Y_{2} \times F_{2}$ as varieties over $Y_{2}$. This finishes the proof of the lemma.

The same argument used in the proof of Lemma 6.4 shows that the following holds.

Lemma 6.5. Let $X$ be a complex projective manifold, and $\varphi: X \rightarrow Y$ be a smooth morphism onto a complex projective manifold. Suppose that $K_{X}$ is pseudo-effective, and suppose that $K_{X / Y} \sim 0$. Suppose furthermore that the irregularity of any fiber of $\varphi$ is zero. Then there exist complex projective manifolds $Y_{1}$ and $F$ as well as a finite étale cover $Y_{1} \rightarrow Y$ such that $Y_{1} \times_{Y} X \cong Y_{1} \times F$ as varieties over $Y_{1}$.

The following is a global version of Reeb stability theorem for regular foliations with numerically trivial canonical class on complex projective manifolds with pseudo-effective canonical divisor. See [HV10, Theorem 3.2] for a somewhat related result.

Proposition 6.6. Let $X$ be a complex projective manifold, let $H$ be an ample divisor on $X$, and let $\mathscr{G} \subset T_{X}$ be a regular foliation with $K_{\mathscr{G}} \equiv 0$. Suppose that $K_{X}$ is pseudo-effective. Suppose furthermore that $\mathscr{G}$ is algebraically integrable, and that one of the following two conditions holds.

(1) The sheaf $\mathscr{G}$ is $H$-semistable and $c_{2}(\mathscr{G}) \equiv 0$.

(2) The sheaf $\mathscr{G}$ is $H$-strongly stable.

Then there exist complex projective manifolds $F$ and $Y$, and a finite étale cover $f: Y \times F \rightarrow X$ such that $f^{-1} \mathscr{G}$ is induced by the projection morphism $Y \times F \rightarrow Y$.

Proof. By [LPT11b, Theorem 5.2], the divisor $K_{\mathscr{G}}$ is a torsion point, and hence, by replacing $X$ with a finite étale cover, if necessary, we may assume without loss of generality that $K_{\mathscr{G}} \sim 0$.

Suppose first that $\mathscr{G}$ is $H$-semistable, and that $c_{2}(\mathscr{G}) \equiv 0$. Applying [LPT11b, Theorem 5.2], we see that there exists a regular foliation $\mathscr{E}$ on $X$ such that $T_{X} \cong \mathscr{G} \oplus \mathscr{E}$. By [Dru16, Lemma 5.8], there exists a finite étale cover $f_{1}: X_{1} \rightarrow X$ such that $f_{1}^{*} \mathscr{G} \cong \mathscr{O}_{X_{1}}^{\oplus r}$, where $r:=\operatorname{rank} \mathscr{G}$. We claim that the neutral component $\operatorname{Aut}\left(X_{1}\right)^{\circ}$ of $\operatorname{Aut}\left(X_{1}\right)$ is an abelian variety. Suppose otherwise. Then, by Chevalley's structure theorem, $\operatorname{Aut}\left(X_{1}\right)^{\circ}$ contains a positive dimensional affine subgroup. Hence, it contains an algebraic subgroup isomorphic to $\mathbb{G}_{m}$ or $\mathbb{G}_{a}$. This implies that $X$ is uniruled, yielding a contradiction since $K_{X}$ is pseudo-effective. Set $A:=\operatorname{Aut}\left(X_{1}\right)^{\circ}$. By [Bri10, Proof of Theorem 1.2, page 10], replacing $X_{1}$ with a further étale cover, if necessary, we may assume that $X_{1} \cong A \times Y$ and that $h^{0}\left(Y, T_{Y}\right)=0$. In particular, we must have $f_{1}^{*} \mathscr{G} \subset T_{A \times Y / Y}$. Thus, there exists a linear foliation $\mathscr{H}$ on $A$ with algebraic leaves such that $f_{1}^{-1} \mathscr{G}=p^{-1} \mathscr{H} \cap T_{A \times Y / Y}$, where $p: X_{1} \rightarrow A$ denotes the projection. Let $F \subset A$ be a leaf of $\mathscr{H}$, and let $B \subset A=\operatorname{Aut}^{\circ}(A)$ be the connected component of the group of elements $a \in A$ such that $a+F=F ; B$ is an algebraic group and its Lie algebra is the kernel of the natural map

$$
\operatorname{Lie}(A) \cong H^{0}\left(A, T_{A}\right) \rightarrow H^{0}\left(F, T_{A \mid F}\right) \rightarrow H^{0}\left(F, \mathscr{N}_{F / A}\right) .
$$




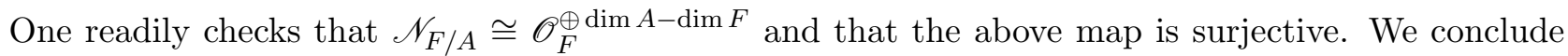
that $B$ is an abelian subvariety of $A$ of dimension $r$. Note that $a+B$ is a leaf of $\mathscr{H}$ for all $a \in A$ since $\mathscr{H}$ is linear. By Poincaré's complete reducibility theorem, there exists an abelian subvariety $C \subset A$ such that the natural morphism $B \times C \rightarrow A$ is an isogeny (see [Mum08, Theorem IV.1]). If $f: B \times C \times Y \rightarrow X$ denotes the induced morphism, then $f^{-1} \mathscr{G}$ is given by the projection $B \times C \times Y \rightarrow$ $C \times Y$, proving Proposition 6.6 in this case.

Suppose from now on that $\mathscr{G}$ is $H$-strongly stable. We may also assume that $c_{2}(\mathscr{G}) \not \equiv 0$.

By the holomorphic version of Reeb stability theorem (see [HV10, Proposition 2.5]), $\mathscr{G}$ is induced by a morphism $\varphi: X \rightarrow Y$ onto a normal projective variety. Applying [HV10, Theorem 2.7], we see that there exists a finite set $\Gamma$ of indices, étale morphisms $f_{\gamma}: X_{\gamma} \rightarrow X$ for all $\gamma \in \Gamma$ as well as smooth projective morphisms with connected fibers $\varphi_{\gamma}: X_{\gamma} \rightarrow Y_{\gamma}$ such that $f_{\gamma}^{-1} \mathscr{G}$ is induced by $\varphi_{\gamma}$ and such that $X=\cup_{\gamma \in \Gamma} f_{\gamma}\left(X_{\gamma}\right)$. The following properties hold in addition. There is a commutative diagram:

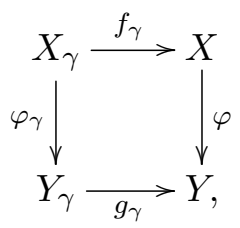

$g_{\gamma}\left(Y_{\gamma}\right)$ is a Zariski open set, and $g_{\gamma}: Y_{\gamma} \rightarrow g_{\gamma}\left(Y_{\gamma}\right)$ is a finite morphism (see Proof of [HV10, Lemma 2.6]). In particular, $X_{\gamma}$ identifies with the normalization of $Y_{\gamma} \times_{Y} X$. Let $g_{1}: Y_{1} \rightarrow Y$ be the normalization of $Y$ in the Galois closure of the compositum of the function fields $\mathbb{C}\left(Y_{\gamma}\right)$. Let $X_{1}$ be the normalization of $Y_{1} \times_{Y} X$, and denote by $f_{1}: X_{1} \rightarrow X$ and $\varphi_{1}: X_{1} \rightarrow Y_{1}$ the natural morphisms. Notice that $\varphi_{1}$ is smooth with connected fibers since $g_{1}: Y \rightarrow Y$ factors through $g_{\gamma}: Y_{\gamma} \rightarrow Y$ over $g_{\gamma}\left(Y_{\gamma}\right)$ and $Y=\cup_{\gamma \in \Gamma} g_{\gamma}\left(Y_{\gamma}\right)$. Finally, let $g_{2}: Y_{2} \rightarrow Y_{1}$ be a desingularization of $Y_{1}$, and set $X_{2}:=Y_{2} \times_{Y_{1}} X_{1}$. Observe that $X_{2}$ is a smooth projective variety, and denote by $f_{2}: X_{2} \rightarrow X_{1}$ and $\varphi_{2}: X_{2} \rightarrow Y_{2}$ the natural morphisms. There is a commutative diagram:

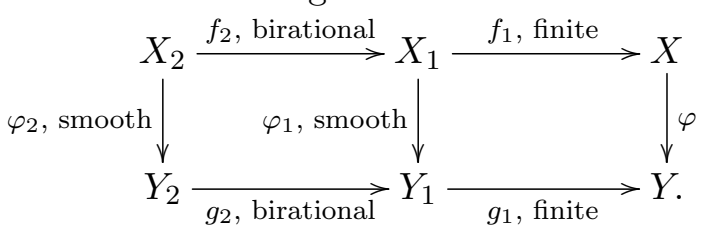

Let $R(\varphi)$ be the ramification divisor of $\varphi$. By Example 2.4 and Lemma 2.6, we have

$$
K_{X_{1} / Y_{1}} \sim f_{1}^{*}\left(K_{X / Y}-R(\varphi)\right) \sim f_{1}^{*} K_{\mathscr{G}} \sim 0 .
$$

This immediately implies that

$$
K_{X_{2} / Y_{2}} \sim f_{2}^{*} K_{X_{1} / Y_{1}} \sim 0
$$

Applying Lemma 6.4 to $\varphi_{2}$, we see that there exist complex projective manifolds $B$ and $S$ as well as a finite étale cover

$$
h: B \times S \rightarrow X_{2}
$$

such that

$$
h^{*} T_{X_{2} / Y_{2}} \cong T_{B \times S / B} .
$$

It follows that $K_{S} \sim 0$. By replacing $S$ with a further étale cover, if necessary, we may assume that $S$ decomposes into the product of an abelian variety and a simply-connected complex projective manifold. In particular, $T_{S}$ is polystable with respect to any polarization. We claim that $S$ is simplyconnected. By assumption, the sheaf $\left(\left(f_{1} \circ f_{2} \circ h\right)^{*} \mathscr{G}\right)^{* *}$ is stable with respect to $\left(f_{1} \circ f_{2} \circ h\right)^{*} H$. Let $C \subset B \times S$ be a smooth complete intersection curve of elements of $\left|\left(f_{1} \circ f_{2} \circ h\right)^{*} m H\right|$ for some sufficiently large integer $m$. Applying [Lan04, Theorem 5.2], we see that $\left(\left(f_{1} \circ f_{2} \circ h\right)^{*} \mathscr{G}\right)^{* *} \mid C$ is stable. On the other hand, by Lemma 2.6, we have $\left(\left(f_{1} \circ f_{2} \circ h\right)^{*} \mathscr{G}^{* *}{ }_{\mid C} \cong T_{B \times S / B}\right.$. This implies that $T_{S}$ is actually stable (with respect to any polarization). If $S$ is a genus one curve, then $\mathscr{G}$ is a line bundle, 
and hence $c_{2}(\mathscr{G}) \equiv 0$, yielding a contradiction. This shows that $S$ is a simply-connected complex projective manifold, proving our claim.

The irregularity of any fiber $F$ of $\varphi_{2}$ is zero because $S$ is simply-connected. Applying Lemma 6.5, we see that there exists a finite étale cover $g_{3}: Y_{3} \rightarrow Y_{2}$ such that

$$
X_{3}:=Y_{3} \times_{Y_{2}} X_{2} \cong Y_{3} \times F
$$

We obtain the following commutative diagram:

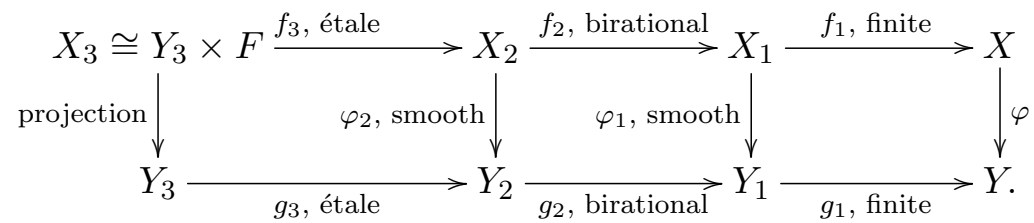

Let $Y_{4}$ be the normalization of $Y_{1}$ in the function field $\mathbb{C}\left(Y_{3}\right)$, and set $X_{4}=Y_{4} \times_{Y_{1}} X_{1}$. This gives another commutative diagram:

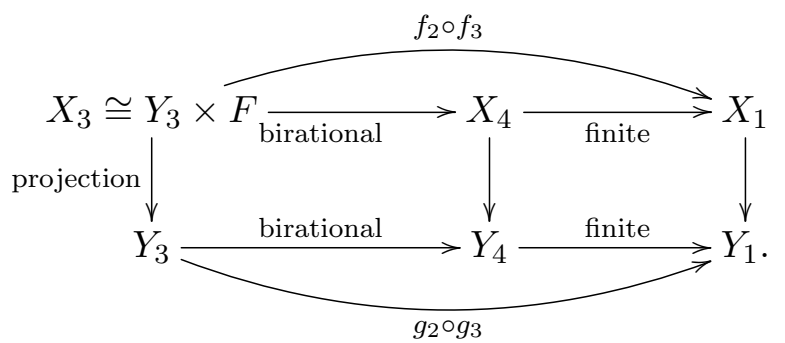

Note that $X_{3}$ identifies with $Y_{3} \times_{Y_{4}} X_{4} \cong X_{3}$. Using the rigidity lemma, we see that the second projection $X_{3} \cong Y_{3} \times F \rightarrow F$ factors into $X_{3} \rightarrow X_{4} \rightarrow F$. This immediately implies that $X_{4} \cong Y_{4} \times F$ over $Y_{4}$.

By replacing $Y_{4}$ with a further finite cover, if necessary, we may assume without loss of generality that the finite cover $Y_{4} \rightarrow Y$ is Galois. In particular, there is a finite group $G$ acting on $Y_{4}$ with quotient $Y$.

The group $G$ also acts on $Y_{4} \times F$ since $Y_{4} \times F$ identifies with the normalization of $Y_{4} \times_{Y} X$. Since $h^{0}\left(F, T_{F}\right)=0, G$ acts on $F$ and its action on $Y_{4} \times F$ is the diagonal action. Let $G_{1}$ denote the kernel of the induced morphism of groups $G \rightarrow \operatorname{Aut}(F)$. By replacing $Y_{4}$ by $Y_{4} / G_{1}, X_{4}$ by $X_{4} / G_{1} \cong\left(Y_{4} / G_{1}\right) \times F$, and $G$ by $G / G_{1}$, if necessary, we may assume without loss of generality that $G \subset \operatorname{Aut}(F)$. Then the quotient map $Y_{4} \times F \rightarrow\left(Y_{4} \times F\right) / G \cong X$ is automatically étale in codimension one, and hence étale by the Nagata-Zariski purity theorem. This finishes the proof of Proposition 6.6 .

The following conjecture is due to Pereira and Touzet (see [PT13]).

Conjecture 6.7. (Pereira-Touzet) Let $\mathscr{G}$ be a regular foliation on a complex projective manifold $X$ with $K_{X}$ pseudo-effective. Suppose that $c_{1}(\mathscr{G}) \equiv 0$ and that $c_{2}(\mathscr{G}) \not \equiv 0$. If $\mathscr{G}$ is stable with respect to some polarization, then it is algebraically integrable.

Building on our recent algebraicity criterion for leaves of algebraic foliations [Dru16, Theorem 1.6], we confirm Conjecture 6.7 in some special cases.

Proposition 6.8. Conjecture 6.7 holds for foliations of rank at most 3.

Proof. We maintain notation and assumptions of Conjecture 6.7. Suppose furthermore that $\mathscr{G}$ has rank $r \leqslant 3$.

Suppose that there exists a finite étale cover $g: Y \rightarrow X$ such that $g^{*} \mathscr{G}$ is not stable. Applying [PT13, Lemma 2.1], we see that there exist non-zero vector bundles $\left(\mathscr{G}_{i}\right)_{i \in I}$, stable with respect to any polarization with $c_{1}\left(\mathscr{G}_{i}\right) \equiv 0$ such that $f^{*} \mathscr{G} \cong \oplus_{i \in I} \mathscr{G}_{i}$. By [Dru16, Proposition 8.1], to prove that $\mathscr{G}$ 
is algebraically integrable, it sufficies to prove that for some $i \in I, \mathscr{G}_{i}$ has algebraic leaves. Therefore, by replacing $X$ with a finite étale cover, if necessary, we may assume that for any finite étale cover $g: Y \rightarrow X, g^{*} \mathscr{G}$ is stable with respect to any polarization.

By replacing $X$ with a further finite étale cover, if necessary, we may also assume that $K_{\mathscr{G}} \sim 0$ by [LPT11b, Theorem 5.2]. Let $H$ be an ample divisor on $X$ such that $c_{2}(\mathscr{G}) \cdot H^{n-2} \neq 0$. By [LPT11b, Theorem 5.2], there exists a regular foliation $\mathscr{E}$ on $X$ such that $T_{X} \cong \mathscr{G} \oplus \mathscr{E}$. The proposition then follows from [Dru16, Theorem 1.6].

We are now in position to prove our main result. Note that Theorem 1.2 is an immediate consequence of Proposition 6.8 and of Theorem 6.9 below.

Theorem 6.9. Let $X$ be a complex projective manifold, and let $\mathscr{G} \subset T_{X}$ be a regular foliation of rank $r$ with $K_{\mathscr{G}} \equiv 0$. Suppose that $K_{X}$ is pseudo-effective. Suppose furthermore that Conjecture 6.7 holds for foliations of rank at most $r$. Then there exist complex projective manifolds $Y$ and $F$, a finite étale cover $f: Y \times F \rightarrow X$, and a regular foliation $\mathscr{H}$ on $Y$ with $c_{1}(\mathscr{H}) \equiv 0$ and $c_{2}(\mathscr{H}) \equiv 0$ such that $f^{-1} \mathscr{G}$ is the pull-back of $\mathscr{H}$.

Proof. Recall from [PT13, Lemma 2.1], that $\mathscr{G}$ is polystable with respect to any polarization. Thus, there is a decomposition $\mathscr{G} \cong \oplus_{i \in I} \mathscr{G}_{i}$ of $\mathscr{G}$ into involutive sub-vector bundles of $T_{X}$ such that $\mathscr{G}_{i}$ is stable with respect to any polarization. Notice that $c_{1}\left(\mathscr{G}_{i}\right) \equiv 0$ for all indices $i \in I$. Notice also that it suffices to prove the theorem for the foliations $\mathscr{G}_{i}$. Therefore, we may assume without loss of generality that $\mathscr{G}$ is stable with respect to any polarization.

Suppose that there exists a finite étale cover $g: Y \rightarrow X$ such that $g^{*} \mathscr{G}$ is not stable. Applying [PT13, Lemma 2.1], we see that there exist non-zero vector bundles $\left(\mathscr{G}_{j}\right)_{j \in J}$, stable with respect to any polarization with $c_{1}\left(\mathscr{G}_{j}\right) \equiv 0$ such that $f^{*} \mathscr{G} \cong \oplus_{j \in J} \mathscr{G}_{j}$. As before, it suffices to prove the theorem for $\mathscr{G}_{j}$ for all indices $j \in J$. Therefore, by replacing $X$ with a further finite étale cover, if necessary, we may assume that for any finite étale cover $g: Y \rightarrow X, g^{*} \mathscr{G}$ is stable with respect to any polarization.

Let $H$ be an ample divisor on $X$. By [BK08, Lemma 40] (see also [Dru16, Lemma 6.20]), there exists a finite étale cover $f: \widetilde{X} \rightarrow X$ such that $\operatorname{Hol}_{\widetilde{x}}\left(f^{*} \mathscr{G}\right)$ is connected, where $\widetilde{x}$ is a point on $\widetilde{X}$. Applying Lemma 6.3, we see that $f^{*} \mathscr{G}$ is strongly stable with respect to $f^{*} H$. If $c_{2}\left(f^{*} \mathscr{G}\right) \equiv 0$, then the statement is obvious. If $c_{2}\left(f^{*} \mathscr{G}\right) \not \equiv 0$, the theorem then follows from Proposition 6.6.

\section{Poisson manifolds - Generalized Bondal conjecture}

In this section, we address the generalized Bondal conjecture. We first recall the basic facts concerning Poisson manifolds.

7.1. (Poisson structures) A (holomorphic) Poisson structure on a complex manifold $X$ is a bivector field $\tau \in H^{0}\left(X, \wedge^{2} T_{X}\right)$, such that the bracket $\{f, g\}:=\langle\tau, d f \wedge d g\rangle$ defines a Lie algebra structure on $\mathscr{O}_{X}$. A Poisson structure defines a skew-symmetric map $\tau^{\sharp}: \Omega_{X}^{1} \rightarrow T_{X}$; the rank of $\tau$ at a point $x \in X$ is the rank of $\tau^{\sharp}(x)$. It is even because $\tau^{\sharp}$ is skew-symmetric. The data of a Poisson structure of $\operatorname{rank} \operatorname{dim} X$ is equivalent to that of a (holomorphic) symplectic structure. In general, we have a partition

$$
X=\bigsqcup_{s \text { even }} X_{s} \quad \text { where } \quad X_{s}:=\left\{x \in X \mid \operatorname{rk} \tau^{\sharp}(x)=s\right\} .
$$

Let $r$ be the generic rank of $\tau$. The generic corank of $\tau$ is defined as $q:=\operatorname{dim} X-r$.

The following conjecture is due to Beauville ([Bea11, Conjecture 5]).

Conjecture 7.2. Let $(X, \tau)$ be a projective Poisson manifold, and let $0 \leqslant s<r$ be an even integer. If $X_{\leq s}:=\bigsqcup_{k \leq s} X_{k}$ is non-empty, it contains a component of dimension $>s$. 
The generalized Bondal conjecture implies that if the degeneracy locus $X \backslash X_{r}$ of $\tau$ is non-empty, then it contains a component of dimension $>r-2$. This is known to be true if $c_{1}(X)^{q+1} \neq 0$ in $H^{q+1}\left(X, \Omega_{X}^{q+1}\right)$ (see [Pol97, Corollary 9.2], and also [Bea11, Proposition 4]).

7.3. (Foliation associated to a Poisson structure) Let $(X, \tau)$ be a Poisson manifold, and let $r$ be the generic rank of $\tau$. The distribution on $X_{r}$ given by Hamiltonian vector fields $\tau^{\sharp}(d f)$ is involutive. We denote by $\mathscr{G} \subset T_{X}$ the corresponding (possibly singular) foliation on $X$. The restriction of $\tau^{\sharp}$ to $X_{r}$ induces a non-degenerate skew-symmetric map $\left(\mathscr{G}_{\mid X_{r}}\right)^{*} \rightarrow \Omega_{X_{r}}^{1} \rightarrow \mathscr{G}_{\mid X_{r}}$. This implies that $\operatorname{det}\left(\mathscr{G}_{\mid X_{r}}\right) \cong \mathscr{O}_{X_{r}}$. Set $\mathscr{N}:=T_{X} / \mathscr{G}$. Notice that $Z(\mathscr{G}) \subset X \backslash X_{r}$. Thus, if the degeneracy locus $X \backslash X_{r}$ of $\tau$ has codimension at least 2 in $X$, then we have

$$
\operatorname{det}(\mathscr{G}) \cong \mathscr{O}_{X} \text { and } \operatorname{det}(\mathscr{N}) \cong \mathscr{O}_{X}\left(-K_{X}\right)
$$

The proof of Theorem 1.3 makes use of the following observation.

Lemma 7.4. Let $(X, \tau)$ be a Poisson manifold, and let $\mathscr{G}$ be the corresponding foliation. Suppose that the degeneracy locus $X \backslash X_{r}$ of $\tau$ has codimension at least 2 in $X$. If $Z(\mathscr{G})$ is empty, then $X=X_{r}$.

Proof. Suppose that $Z(\mathscr{G})=\emptyset$. Then $\mathscr{G}$ is a vector bundle with $\operatorname{det}(\mathscr{G}) \cong \mathscr{O}_{X}$. It follows that the composed morphism $\mathscr{G}^{*} \rightarrow \Omega_{X}^{1} \rightarrow \mathscr{G}$ must be an isomorphism. The lemma easily follows.

In view of the generalized Bondal conjecture, one may ask the following.

Conjecture 7.5. Let $X$ be a complex projective manifold, and let $\mathscr{G} \subset T_{X}$ be foliation of codimension $q$ on $X$ with numerically trivial canonical class. If the singular locus $Z(\mathscr{G})$ of $\mathscr{G}$ is non-empty, it contains a component of codimension $\leqslant q+1$.

The statement is obviously true in the case $q=0$. It is also known to be true if $q=1$ by [LPT11a, Corollary 4.7] (see also Proposition 5.1).

Remark 7.6. In the setup of Conjecture 7.5, if $Z(\mathscr{G})$ is non-empty, then $K_{X}$ is not pseudo-effective by [LPT11b, Theorem 5.4].

Theorem 7.7. Let $X$ be a complex projective manifold, and let $\mathscr{G} \subset T_{X}$ be foliation of codimension $q \leqslant 2$ on $X$ with numerically trivial canonical class. If the singular locus $Z(\mathscr{G})$ of $\mathscr{G}$ is non-empty, it contains a component of codimension $\leqslant q+1$.

Proof. Suppose that $Z(\mathscr{G})$ is non-empty. Applying [LPT11b, Theorem 5.4], we see that $K_{X}$ is not nef. The theorem then follows from Proposition 5.1 and Theorem 5.2.

Proof of Theorem 1.3. We maintain notation and assumptions of Theorem 1.3. The statement is easy if $r=\operatorname{dim} X$. Suppose now that $r \in\{\operatorname{dim} X-2, \operatorname{dim} X-1\}$, and let $\mathscr{G}$ be the natural foliation associated to $\tau$. Suppose moreover that $X \backslash X_{r}$ is non-empty and has codimension at least 2 in $X$. From Lemma 7.4, we conclude that $Z(\mathscr{G})$ is non-empty as well. The statement then follows from Theorem 7.7 above applied to $\mathscr{G}$ using the fact that $Z(\mathscr{G}) \subset X \backslash X_{r}$.

\section{References}

[AD13] Carolina Araujo and Stéphane Druel, On Fano foliations, Adv. Math. 238 (2013), 70-118. MR-3033631

[AD14]_, On codimension 1 del Pezzo foliations on varieties with mild singularities, Math. Ann. 360 (2014), no. 3-4, 769-798. MR-3273645 
[And85] Tetsuya Ando, On extremal rays of the higher-dimensional varieties, Invent. Math. 81 (1985), no. 2, 347-357. MR-0799271

[Ati57] M.F. Atiyah, Complex analytic connections in fibre bundles, Trans. Amer. Math. Soc. 85 (1957), 181-207. MR-0086359

[AW97] Marco Andreatta and Jarosław A. Wiśniewski, A view on contractions of higher-dimensional varieties. In: Algebraic geometry - Santa Cruz 1995, pp. 153-183, Proc. Sympos. Pure Math., vol. 62, Amer. Math. Soc., Providence, RI, 1997. MR-1492522

[BB70] Paul F. Baum and Raoul Bott, On the zeros of meromorphic vector-fields. In: Essays on Topology and Related Topics (Mémoires dédiés à Georges de Rham), pp. 29-47, Springer, New York, 1970, pp. 29-47. MR-0261635

[Bea77] Arnaud Beauville, Variétés de Prym et jacobiennes intermédiaires, Ann. Sci. École Norm. Sup. (4) 10 (1977), no. 3, 309-391. MR-0472843

[Bea83] _ Variétés Kähleriennes dont la première classe de Chern est nulle, J. Differential Geom. 18 (1983), no. 4, 755-782 (1984). MR-0730926

[Bea11] _ Holomorphic symplectic geometry: a problem list. In: Complex and differential geometry, pp. 49-63, Springer Proc. Math., vol. 8, Springer, Heidelberg, 2011. MR-2964467

[BK08] V. Balaji and János Kollár, Holonomy groups of stable vector bundles, Publ. Res. Inst. Math. Sci. 44 (2008), no. 2, 183-211. MR-2426347

[Bri10] Michel Brion, Some basic results on actions of nonaffine algebraic groups. In: Symmetry and spaces, pp. 1-20, Progr. Math., vol. 278, Birkhäuser Boston, Inc., Boston, MA, 2010. MR-2562620

[Dru16] Stéphane Druel, A decomposition theorem for singular spaces with trivial canonical class of dimension at most five, preprint, 2016. arXiv:1606.09006

[Dru17]__ Regular foliations on weak fano manifolds, Ann. Fac. Sci. Toulouse Math. (6) 26 (2017), no. 1, 207-217.

[GP11] Philippe Gille and Patrick Polo (eds.), Schémas en groupes (SGA 3). Tome I. Propriétés générales des schémas en groupes, Séminaire de Géométrie Algébrique du Bois Marie 196264. A seminar directed by M. Demazure and A. Grothendieck with the collaboration of M. Artin, J.-E. Bertin, P. Gabriel, M. Raynaud and J-P. Serre. Revised and annotated edition of the 1970 French original, Documents Mathématiques (Paris), 7, Société Mathématique de France, Paris, 2011. MR-2867621

[HL97] D. Huybrechts and M. Lehn, The geometry of moduli spaces of sheaves, Aspects of Mathematics, E31, Friedr. Vieweg \& Sohn, Braunschweig, 1997. MR-1450870

[HV10] Jun-Muk Hwang and Eckart Viehweg, Characteristic foliation on a hypersurface of general type in a projective symplectic manifold, Compos. Math. 146 (2010), no. 2, 497-506. MR2601637

[Jou79] J.P. Jouanolou, Équations de Pfaff algébriques, Lecture Notes in Mathematics, vol. 708, Springer, Berlin, 1979. MR-0537038

[Kem92] George R. Kempf, Pulling back bundles, Pacific J. Math. 152 (1992), no. 2, 319-322. MR1141798 
[KMM87] Yujiro Kawamata, Katsumi Matsuda, and Kenji Matsuki, Introduction to the minimal model problem. In: Algebraic geometry, Sendai, 1985, pp. 283-360, Adv. Stud. Pure Math., vol. 10, North-Holland, Amsterdam, 1987. MR-0946243

[Kol86] János Kollár, Higher direct images of dualizing sheaves. I, Ann. of Math. (2) 123 (1986), no. 1, 11-42. MR-0825838

[Lan04] Adrian Langer, Semistable sheaves in positive characteristic, Ann. Math. (2) 159 (2004), no. 1, 251-276. MR-2051393

[LPT11a] Frank Loray, Jorge Vitório Pereira, and Frédéric Touzet, Singular foliations with trivial canonical class, preprint, 2011. arXiv:1107.1538v3

[LPT11b] _ _ Singular foliations with trivial canonical class, preprint, 2015. arXiv:1107.1538v4

[Mum08] David Mumford, Abelian varieties. With appendices by C. P. Ramanujam and Yuri Manin. Corrected reprint of the second (1974) edition. Tata Inst. Fund. Res. Stud. Math., vol. 5.. Published for the Tata Institute of Fundamental Research, Bombay; by Hindustan Book Agency, New Delhi, 2008. MR-2514037

[NS65] M. S. Narasimhan and C.S. Seshadri, Stable and unitary vector bundles on a compact Riemann surface, Ann. Math. (2) 82 (1965), 540-567. MR-0184252

[Pol97] A. Polishchuk, Algebraic geometry of Poisson brackets, J. Math. Sci. (New York) 84 (1997), no. 5, 1413-1444, Algebraic geometry, 7. MR-1465521

[PT13] Jorge Vitório Pereira and Frédéric Touzet, Foliations with vanishing Chern classes, Bull. Braz. Math. Soc. (N.S.) 44 (2013), no. 4, 731-754. MR-3167130

[ST71] Yum-tong Siu and Günther Trautmann, Gap-sheaves and extension of coherent analytic subsheaves, Lecture Notes in Mathematics, vol. 172, Springer-Verlag, Berlin-New York, 1971. MR-0287033

[Tou08] Frédéric Touzet, Feuilletages holomorphes de codimension un dont la classe canonique est triviale, Ann. Sci. Éc. Norm. Supér. (4) 41 (2008), no. 4, 655-668. MR-2489636

[Tou10] Frédéric Touzet, Structure des feuilletages Kähleriens en courbure semi-négative, Ann. Fac. Sci. Toulouse, Math. (6) 19 (2010), no. 3-4, 865-886. MR-2790821

[Wiś91] J.A. Wiśniewski, On contractions of extremal rays of Fano manifolds, J. Reine Angew. Math. 417 (1991), 141-157. MR-1103910 